\title{
Florística vascular da Floresta Ombrófila Densa Altomontana de quatro serras no Paraná
}

\author{
Maurício Bergamini Scheer ${ }^{1,2,4}$ \& Alan Yukio Mocochinski ${ }^{3}$ \\ ${ }^{1}$ Programa de Pós-Graduação em Engenharia Florestal, Universidade Federal do Paraná - UFPR, \\ Av. Pref. Lothário Meissner, 900, Jardim Botânico, Campus III \\ CEP 80210-170, Curitiba, PR, Brasil \\ ${ }^{2}$ Assessoria de Pesquisa e Desenvolvimento APD/DMA - SANEPAR, \\ Rua Engenheiros Rebouças, 1376, Rebouças, CEP 80215-900, Curitiba, PR, Brasil \\ ${ }^{3}$ Coordenação de Aperfeiçoamento de Pessoal de Nivel Superior-CAPES/MEC \\ Esplanada dos Ministérios, Bloco L, Anexo II, Sala 204, CEP 70359-970, Brasilia, DF, Brasil \\ ${ }^{4}$ Autor para correspondência: Maurício Bergamini Scheer, e-mail: mauriciobs@sanepar.com.br
}

SCHEER, M.B. \& MOCOCHINSKI, A.Y. Floristic composition of four tropical upper montane rain forests in Southern Brazil. Biota Neotrop., 9(2): http://www.biotaneotropica.org.br/v9n2/en/ abstract?article+bn00609022009.

\begin{abstract}
The Cloud Forests have very important environmental functions, among them, the maintenance and protection of the origin of the watersheds and the carbon stocks in its biomass and into the soil, besides its biodiversity and endemism. Despite still exist considerable remnants of primary cloud forests there are few studies that listed species that occur in these ecosystems. The aim of this study was to characterize the floristic composition of four areas of the Upper Montane Rain Forest of the "Serra do Mar" in the state of Paraná and to compare it with other cloud forests in southern and southeastern Brazil. A total of 346 vascular species were detected. They comprised 87 families including 72 angiosperms (288 species), 14 pteridophytes (57 species) and one gymnosperm. The species richest families were Myrtaceae (34 species; 10\% of total), Asteraceae (30; 9\%), Orchidaceae $(29 ; 8 \%)$, Rubiaceae $(17 ; 5 \%)$, Melastomataceae $(16 ; 5 \%)$, Poaceae $(12 ; 3 \%)$ and Bromeliaceae $(11 ; 3 \%)$. The Serra do Ibitiraquire presented the largest area of cloud forests and the highest species richness (231 species). Of the 346 species found in typical cloud forests, 231 species were classified as typical, 41 as transitionals from high altitude grasslands and 68 as transitionals from lower montane forests. Similarities between the studied areas and other Brazilian cloud forests were low (cluster analyses and Sörensen indexes). Besides the geological, geomorphological, pedological and forest structural differences, the conservation status, the lower influence of "Mixed Ombrophyllous forests" (Araucaria Moist forests) species, pioneer and lower montane species, justify this lower similarity.

Keywords: atlantic rain forest, sea mountain range, tropical/subtropical montane cloud forest, upper montane dense ombrophyllous forest.
\end{abstract}

SCHEER, M.B. \& MOCOCHINSKI, A.Y. Florística vascular da Floresta Ombrófila Densa Altomontana de quatro serras no Paraná. Biota Neotrop., 9(2): http://www.biotaneotropica.org.br/v9n2/pt/ abstract?article+bn00609022009.

Resumo: A Floresta Ombrófila Densa Altomontana é uma formação responsável por importantes funções ambientais, entre elas a proteção e manutenção dos fluxos hídricos de cabeceiras de bacias hidrográficas, o estoque de carbono na sua biomassa e na do solo, além da sua biodiversidade e seu elevado endemismo. Apesar de ainda existirem remanescentes primários significativos dessas florestas, apenas alguns estudos descreveram sua estrutura arbórea. O presente trabalho tem o objetivo de listar e comparar a florística vascular de quatro serras representativas da Floresta Ombrófila Densa Altomontana no Paraná e de comparar a florística arbórea das florestas do presente estudo com a de outras florestas semelhantes nas regiões sul e sudeste do Brasil. Foram detectadas 346 espécies vegetais vasculares, pertencentes a 176 gêneros e a 87 famílias, sendo 72 angiospermas (288 espécies), 14 pteridófitas (57 espécies) e 1 gimnosperma. A família com maior riqueza específica foi Myrtaceae, com 34 espécies (10\% do total), seguida por Asteraceae (30; 9\%), Orchidaceae (29; 8\%), Rubiaceae $(17 ; 5 \%)$, Melastomataceae (16; 5\%), Poaceae (12;3\%) e Bromeliaceae (11; 3\%). A composição florística arbórea das florestas altomontanas da Serra do Mar paranaense apresentou a menor similaridade entre as três grandes serras comparadas, com índices um pouco maiores com as florestas altomontanas da região de Aparados da Serra Geral (SC) e menores com a Serra da Mantiqueira, sudeste do Brasil (SP, RJ e MG). Além de diferenças geológicas, geomorfológicas, pedológicas e fitofisionômicas, as diferenças florísticas encontradas nas florestas altomontanas da Serra do Mar do Paraná em relação às demais serras comparadas pode também ser explicada pela melhor conservação dos trechos amostrados e pela baixa influência de elementos de outros tipos vegetacionais próximos (Floresta Ombrófila Mista, Floresta Ombrófila Densa Montana e vegetação secundária).

Palavras-chave: floresta atlântica, floresta ombrófila densa altomontana, floresta nebular tropical/subtropical, serra do mar. 


\section{Introdução}

Os ecossistemas altomontanos (Floresta Ombrófila Densa Altomontana e Refúgios Vegetacionais Altomontanos; Veloso et al. 1991) são ambientes singulares, que recebem um aporte adicional de água por estarem situados nos patamares altimétricos superiores das montanhas, onde as nuvens são mais freqüentes (Hamilton et al. 1995). A retenção hídrica desses ecossistemas é ainda maior devido à redução da radiação solar e da evapotranspiração (Hamilton et al. 1995, Bruijnzeel \& Proctor 1995). As menores temperaturas em altitudes elevadas também diminuem as taxas de decomposição da biomassa, causando um maior acúmulo de matéria orgânica nos solos. Essa característica indica altos potenciais de fixação de carbono e de retenção hídrica (Bruijnzeel 2000). Aliado à sua importância hidrológica, principalmente na proteção e manutenção de cabeceiras das bacias hidrográficas, está sua importância para a diversidade biológica, uma vez que comporta altos níveis de endemismo animal e vegetal (Hamilton et al. 1995).

No Brasil, as florestas altomontanas, também chamadas de florestas nebulares, florestas altimontanas ou matinhas nebulares, foram descritas inicialmente por Dusén (1955) na região sudeste e por Klein (1980) na região sul. Estudos recentes em florestas altomontanas em condições diferentes (ecótonos, florestas deciduais e semideciduais e ombrófilas mistas) foram realizados por Meira Neto et al. (1989), Fontes (1997), França \& Stehmann (2004), Oliveira-Filho et al. (2004) e Carvalho et al. (2005), no estado de Minas Gerais e por Falkenberg \& Voltolini (1995) e Falkenberg (2003) em Santa Catarina e no Rio Grande do Sul. Também nos últimos anos, descrições mais detalhadas da Floresta Ombrófila Densa Altomontana no Paraná foram realizadas pelos trabalhos de Bolòs et al. (1991), Roderjan (1994), Rocha (1999), Koehler (2001), Portes et al. (2001), Petean (2002) e Koehler et al. (2002).

No Paraná, a Floresta Ombrófila Densa Altomontana ocorre, em média, a partir dos $1.200 \mathrm{~m}$ s.n.m. e caracteriza-se por significativas diferenças estruturais quando comparadas às florestas de patamares altimétricos mais baixos. Dentre essas diferenças, as mais marcantes são a menor altura das árvores (em torno de $4 \mathrm{~m}$ ); a maior densidade de fustes; formação de apenas um estrato com copas entremeadas que formam um dossel bastante denso e com ausência de árvores emergentes; troncos e ramos retorcidos; folhas pequenas e freqüentemente coriáceas e a abundância de epífitas. Além disso, com a elevação da altitude, percebe-se uma gradativa diminuição da riqueza de espécies, reflexo do aumento do grau de adversidade ambiental (Roderjan 1994). Este tipo vegetacional apresenta um elevado grau de endemismo, decorrente de pressões de seleção singulares.

Os ecossistemas altomontanos, por se localizarem em áreas de difícil acesso, em geral apresentam menores potenciais de ocupação imobiliária, de produção agropecuária e de exploração madeireira. Porém, muitos remanescentes vêm sendo descaracterizados devido à introdução de espécies exóticas, à exploração de madeira e de recursos não madeiráveis, às queimadas utilizadas nas atividades agrícolas e silviculturais, ao turismo desordenado, à extração de plantas ornamentais e medicinais, à caça, à mineração, à construção de estradas e à instalação de torres de telecomunicação (Doumenge et al. 1995, Hamilton et al. 1995, Vitousek 1998). Bruijnzeel (2000) citou diversos autores que apontaram a possibilidade do aquecimento global elevar o patamar altimétrico de condensação das nuvens e, possivelmente, diminuir as oportunidades dos ecossistemas interceptarem a água presente nelas. Em diversas regiões, a taxa de desaparecimento das florestas em montanhas excede às das áreas pluviais tropicais de menores altitudes (Hamilton et al. 1995). De acordo com a FAO, a perda anual de florestas em montanhas tropicais, no período entre $1981 \mathrm{e}$ 1990 , foi de $1,1 \%$ comparado com $0,8 \%$ para as demais florestas dos trópicos (Doumenge et al. 1995). A degradação desses ecossistemas ainda é agravada pela sua baixa resiliência (Hamilton et al. 1995).

Falkenberg \& Voltolini (1995) salientaram a falta de conhecimento a respeito da diversidade de espécies e a necessidade de pesquisas biológicas básicas e inventários como pré-requisito para ações para a conservação e restauração desses ambientes. A maior parte do conhecimento científico sobre os ecossistemas altomontanos tropicais concentra-se nas montanhas da América Central e noroeste da América do Sul. Estudos florísticos sobre tais ecossistemas podem apontar índices de diversidade, espécies novas, raras, endêmicas, indicadoras de ambientes ainda bem conservados, subsidiar estudos fitogeográficos e o fortalecimento de estratégias de conservação da diversidade biológica e da qualidade ambiental.

No presente trabalho foram avaliados trechos de Floresta Ombrófila Densa Altomontana em quatro serras no Estado do Paraná (altitudes entre 950 e $1.850 \mathrm{~m}$ ), pertencentes ao Complexo Serra do Mar, com os objetivos de: 1) listar as espécies da flora vascular; 2) comparar a florística vascular das quatro serras entre si; 3) comparar a florística arbórea das florestas do presente estudo com a de outras florestas ombrófilas acima dos 1.380 e 1.800 m de altitude, respectivamente nas regiões sul e sudeste do Brasil.

\section{Material e Métodos}

\section{1. Áreas de estudo}

O conjunto de montanhas das serras do Mar e da Mantiqueira constitui a mais destacada feição orográfica da borda atlântica do continente sul-americano. A Serra do Mar se estende do Rio de Janeiro ao norte de Santa Catarina, onde se desfaz em cordões de serras paralelas e montanhas isoladas drenadas diretamente para o mar, sobretudo pela bacia do rio Itajaí (Almeida \& Carneiro, 1998).

No Paraná, a Serra do Mar constitui em uma zona limítrofe entre o litoral e o planalto meridional. Pelo lado continental, esta região se eleva de 500 a 1.000 m sobre o nível médio do planalto e pelo lado oriental pode se elevar a mais de 1.800 m sobre o nível do mar, sendo este mais escarpado. É dividida em vários maciços, escarpas e restos de planalto profundamente dissecados, formando primordialmente um arco granitóide com concavidade voltada para o leste. Os vários blocos diminuem suas elevações de nordeste para sudoeste com denominações regionais especiais de serras: do Capivari Grande, da Virgem Maria, do Ibitiraquire, da Graciosa, da Farinha Seca, do Marumbi, da Igreja, dos Castelhanos, do Araçatuba, da Baitaca, da Pedra Branca do Araraquara, da Prata, das Canavieiras, entre outras (Maack 2002, Bigarella 1978). Entre estes maciços, a Serra dos Órgãos paranaense (ou Ibitiraquire) possui as maiores elevações da região sul do país, tendo como seus pontos culminantes os picos Paraná, com $1.887 \mathrm{~m}$ s.n.m., e Caratuva, com 1.850 m s.n.m.

O clima das florestas altomontanas da Serra do Mar paranaense é classificado como Cfb, segundo Köppen, sendo subtropical, sempre úmido e com a temperatura média do mês mais frio abaixo de $18{ }^{\circ} \mathrm{C}$ e superior a $-3{ }^{\circ} \mathrm{C}$ e a média do mês mais quente inferior a $22{ }^{\circ} \mathrm{C}$ (Roderjan 1994). Roderjan \& Grodski (1999) observaram temperatura mínima absoluta de $-5^{\circ} \mathrm{C}$, média anual de $13,4{ }^{\circ} \mathrm{C}$ e máxima absoluta de $30{ }^{\circ} \mathrm{C}$ para patamares altomontanos a $1.385 \mathrm{~m}$ s.n.m., em ambiente florestal, no Morro do Anhangava, no município de Quatro Barras, Paraná. As precipitações na Serra do Mar são bem distribuídas ao longo do ano e apresentam grande variação em função da topografia local. Medições na região litorânea ultrapassam $2.000 \mathrm{~mm}$ anuais e nas encostas da serra os valores chegam a $3.500 \mathrm{~mm}$ (Maack 2002).

A Floresta Ombrófila Densa Atlântica cobre a maior extensão das montanhas, atingindo, em sua formação altomontana, as porções mais 
elevadas das encostas e vales, acima de 1.800 m s.n.m. Em alguns trechos, nas encostas a oeste, ocorre o ecótono entre esta e a Floresta Ombrófila Mista Montana, a aproximadamente 1.100 m s.n.m., com ocorrência de Araucaria angustifolia. Já nas porções mais elevadas das montanhas, passam a ocorrer os campos altomontanos.

Foram amostradas quatro sub-serras, entre as coordenadas $26^{\circ} 00^{\prime} \mathrm{S}$ e $49^{\circ} 30^{\prime} \mathrm{W}$, e $25^{\circ} 00^{\prime} \mathrm{S}$ e $48^{\circ} 00^{\prime} \mathrm{W}$, denominadas regionalmente de: Serra do Ibitiraquire, Serra da Igreja, Serra da Prata e Serra Gigante (Figura 1). A escolha das áreas de estudo levou em consideração a representatividade da área de ocorrência dessa formação no Paraná. Os solos das áreas amostradas são predominantemente Neossolos Litólicos, Organossolos não hidromórficos, podendo ocorrer Cambissolos e Argissolos Vermelho-Amarelos (Roderjan et al. 2002, Rocha 1999). As florestas altomontantas nos trechos estudados estão inseridas em diferentes unidades de conservação (Tabela 1).

\section{Levantamento florístico}

O levantamento florístico foi realizado entre agosto de 2002 e agosto de 2004, com fases de campo mensais. O esforço amostral variou de acordo com a extensão das áreas de estudo (diferente número e tamanho de montanhas e picos amostrados, conforme Tabela 1) e com fatores logísticos, relacionados principalmente com a dificuldade de acesso aos trechos de ocorrência da formação. Foram realizados 55 dias de levantamento florístico com coletas aleatórias ao longo de trilhas, bem como locais mais isolados, empreendidos concomitantemente com o levantamento da estrutura arbórea da formação ( 25 parcelas de $10 \times 10 \mathrm{~m}$ em cada serra), cujos resultados ainda não foram publicados.
Em cada fase de campo procedeu-se a coleta das espécies vegetais vasculares encontradas férteis na área de ocorrência da Floresta Ombrófila Densa Altomontana. Uma vez que a transição entre as formações de floresta de um patamar altitudinal para outro é dado por um gradiente de mudanças florísticas e estruturais, a amostragem do presente trabalho evitou áreas de transição entre as formações Montana e a Altomontana. Assim, as coletas foram efetuadas apenas em trechos de floresta com características estruturais e fisionômicas tipicamente altomontanas, tais como: presença de apenas um estrato arbóreo, com porte reduzido ( 3 a $7 \mathrm{~m}$ de altura) e com troncos com muitas ramificações tortuosas, densamente cobertas por epífitas avasculares, as quais foram classificadas como florestas altomontanas típicas (Figura 2d). Segundo revisão bibliográfica de Stadtmüller (1987), tais considerações se enquadram nas descrições de formações de florestas nebulares "Cloud Forests" localizadas nos patamares superiores das montanhas, denominadas "Elfin Woodlands" ou "Dwarf Cloud Forests". Da mesma forma, não foram amostrados trechos de transição entre a floresta altomontana e os campos altomontanos. Naturalmente, espécies normalmente ocorrentes nessas formações vizinhas eventualmente foram detectadas nos trechos estudados, sendo que para o presente estudo foram classificadas como transicionais e compõem a diversidade florística da formação. A integridade dos trechos estudados foi outro critério para a amostragem. Procederam-se coletas apenas em trechos de florestas em estágio primário, sendo que áreas alteradas e de sucessão secundária não foram amostradas.

A determinação das espécies foi realizada com o uso de chaves de identificação, comparação com material de herbários e confirmações com especialistas. Utilizou-se a classificação taxonômica proposta por APG II (2003) para o reconhecimento das famílias de angiospermas, e

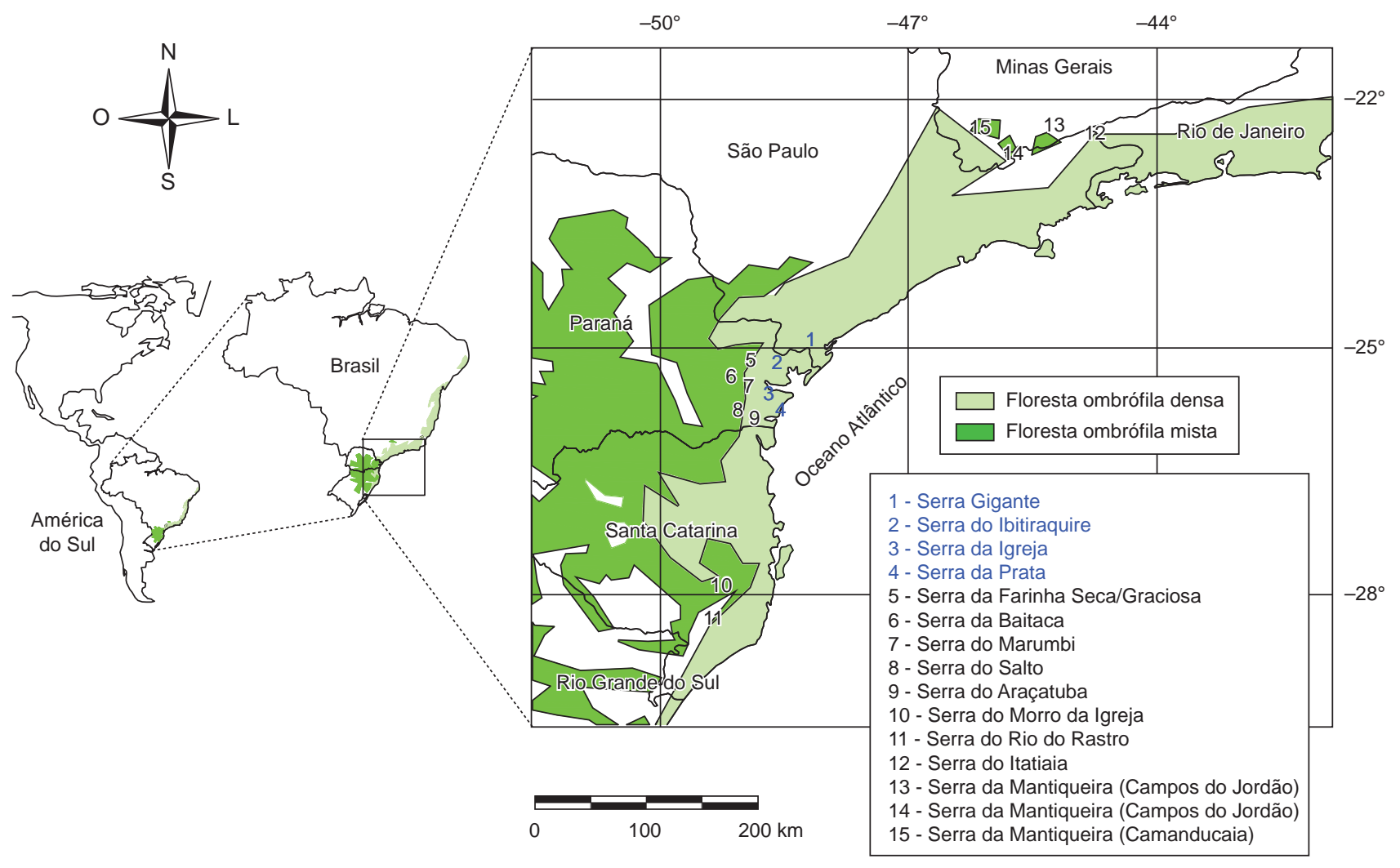

Figura 1. Localização das áreas de estudo no estado do Paraná. Adaptado de IBGE 2004.

Figure 1. Location of the studied areas in the state of Paraná, Southern Brazil. Adapted from IBGE 2004. 
Tabela 1. Características das áreas amostradas na Serra do Mar no Paraná. APA - Área de Proteção Ambiental; PE - Parque Estadual; PN - Parque Nacional Table 1. Characteristics of the studied areas in Sea Mountain Range in the state of Paraná, Southern Brazil; APA - Environmental Protection Area; PE - State Park; PN - National Park

\begin{tabular}{|c|c|c|c|c|c|c|}
\hline Serra & $\begin{array}{c}\text { Ponto } \\
\text { Culminante } \\
\text { (m s.n.m.) } \\
\end{array}$ & $\begin{array}{c}\text { Localização } \\
\text { (Municípios) }\end{array}$ & Litologia* & $\begin{array}{c}\text { Coordenadas } \\
\text { UTM, Datum } \\
\text { SAD 69, Fuso 22 } \\
\end{array}$ & Características & $\begin{array}{c}\text { Proteção por } \\
\text { UC }\end{array}$ \\
\hline do Ibitiraquire & 1.887 & $\begin{array}{l}\text { Campina } \\
\text { Grande do Sul } \\
\text { e Antonina }\end{array}$ & $\begin{array}{l}\text { Álcali- } \\
\text { Granitos } \\
\text { (Granito } \\
\text { Graciosa) }\end{array}$ & $\begin{array}{r}719.000 \mathrm{E} \\
7.205 .000 \mathrm{~N}\end{array}$ & $\begin{array}{l}\text { Trecho mais extenso e de maior } \\
\text { gradiente altitudinal de florestas } \\
\text { altomontanas. Existem } 13 \\
\text { montanhas com mais de } 1.500 \mathrm{~m} \\
\text { s.n.m. (10 montanhas amostradas). }\end{array}$ & $\begin{array}{l}\text { PE's do Pico } \\
\text { Paraná e } \\
\text { Roberto Ribas } \\
\text { Lange }\end{array}$ \\
\hline da Igreja & 1.376 & $\begin{array}{l}\text { Morretes, } \\
\text { São José dos } \\
\text { Pinhais e } \\
\text { Guaratuba }\end{array}$ & $\begin{array}{c}\text { Álcali- } \\
\text { Granitos } \\
\text { (Granito Serra } \\
\text { da Igreja) }\end{array}$ & $\begin{array}{r}715.000 \mathrm{E} \\
7.164 .000 \mathrm{~N}\end{array}$ & $\begin{array}{c}\text { Florestas altomontanas ocorrem } \\
\text { a partir de } 1.200 \mathrm{~m} \text { s.n.m.. Possui } \\
\text { topos aplainados ( } 3 \text { montanhas } \\
\text { amostradas). }\end{array}$ & $\begin{array}{c}\text { APA de } \\
\text { Guaratuba }\end{array}$ \\
\hline da Prata & 1.502 & $\begin{array}{l}\text { Morretes, } \\
\text { Paranaguá e } \\
\text { Guaratuba }\end{array}$ & $\begin{array}{c}\text { Complexo } \\
\text { Gnáissico- } \\
\text { Migmatítico } \\
\text { (Suíte } \\
\text { Granítica } \\
\text { Foliada) }\end{array}$ & $\begin{array}{r}725.000 \mathrm{E} \\
7.166 .000 \mathrm{~N}\end{array}$ & $\begin{array}{c}\text { Florestas altomontanas ocorrem } \\
\text { a partir de } 1.200 \text { m s.n.m.. Serra } \\
\text { isolada na planície litorânea, sem } \\
\text { contato com o planalto (uma } \\
\text { montanha amostrada). }\end{array}$ & $\begin{array}{c}\text { PN Saint } \\
\text { Hilaire-Lange }\end{array}$ \\
\hline Gigante & 1.069 & $\begin{array}{l}\text { Guaraqueçaba } \\
\text { (PR) e } \\
\text { Cananéia (SP) }\end{array}$ & $\begin{array}{c}\text { Complexo } \\
\text { Gnáissico- } \\
\text { Migmatítico } \\
\text { (Suíte } \\
\text { Granítica } \\
\text { Foliada) }\end{array}$ & $\begin{array}{r}786.000 \mathrm{E} \\
7.216 .000 \mathrm{~N}\end{array}$ & $\begin{array}{c}\text { Florestas altomontanas ocorrem a } \\
\text { partir de } 950 \text { m s.n.m.. Serra isolada } \\
\text { na planície litorânea, sem contato } \\
\text { com o planalto. (5 montanhas } \\
\text { amostradas). }\end{array}$ & $\begin{array}{l}\text { APA de } \\
\text { Guaraqueçaba } \\
\text { e PE de } \\
\text { Jacupiranga } \\
\text { (SP) }\end{array}$ \\
\hline
\end{tabular}

*de acordo com PRÓ-ATLÂNTICA, 2002.

a proposta por Tryon e Tryon (1982) para as pteridófitas. O material de referência foi incorporado ao acervo do Museu Botânico Municipal de Curitiba (MBM) e as duplicatas doadas ao Herbário do Departamento de Botânica da Universidade Federal do Paraná (UPCB) e ao Herbário da Escola de Florestas de Curitiba (EFC).

Para a comparação da florística vascular das florestas altomontanas amostradas e da florística arbórea dessas com a de outras florestas ocorrentes acima dos $1.380 \mathrm{~m}$ de altitude na região sul (PR e SC) e acima dos $1.800 \mathrm{~m}$ na região sudeste (SP, RJ e MG) do Brasil, foram calculados os índices de similaridade de Sörensen (Brower \& Zar 1984) e realizadas análises de agrupamentos (Cluster) envolvendo presença e ausência de espécies. Tais florestas foram escolhidas para análise por serem descritas como ombrófilas e altomontanas, ocorrentes em importantes complexos montanhosos do sul e sudeste do Brasil: Aparados da Serra Geral, Serra do Mar e Serra da Mantiqueira (Tabela 2). Nas análises envolvendo só as quatro serras amostradas pelo presente trabalho, foram consideradas determinações em nível de espécie, de gênero e também morfotipos. Já para as análises envolvendo dados de outros trabalhos (florística arbórea), foram consideradas somente determinações em nível de espécie. $\mathrm{O}$ agrupamento foi realizado pelo método de Ward (variância mínima) com a utilização da distância euclidiana quadrática como medida métrica. Segundo Valentin (2000), tal método é considerado muito eficiente em estudos ecológicos, pois resulta em dendrogramas bem representativos da realidade.

\section{Resultados e Discussão}

\section{Florística vascular das serras amostradas no presente trabalho}

Nos quatro trechos estudados na Serra do Mar paranaense, foram encontradas 346 espécies vegetais vasculares (Tabela 3 ), pertencentes a 176 gêneros e a 87 famílias, sendo 72 angiospermas (288 espécies), 14 pteridófitas (57 espécies) e 1 gimnosperma.

Quanto à forma de vida das espécies registradas, 30,6\% (106 espécies) são árvores, outros 30,6\% (106 spp.) são herbáceas, $15,6 \%$ (54) são epífitas, $14,7 \%$ (51) são arbustos, 7,5\% (26) são trepadeiras e $0,9 \%$ (3) são hemiparasitas. As 106 espécies arbóreas pertencem a 37 famílias, sendo a de maior riqueza específica Myrtaceae (10 gen., 34 spp.), seguida por Lauraceae (4 gen., 9 spp.), Rubiaceae (4 gen., 6 spp.) e por Aquifoliaceae (1 gen. 6 spp.). Os dados são similares à compilação de Oliveira-Filho \& Fontes (2000), em que Myrtaceae, Melastomataceae, Lauraceae e Rubiaceae foram consideradas famílias com maior número de espécies arbóreas em florestas ombrófilas atlânticas de patamares montanos e altomontanos. Koehler et al. (2001) listaram 55 espécies pertencentes a 24 famílias em uma compilação de resultados de estudos sobre a estrutura arbórea de seis áreas de florestas altomontanas no Paraná.

A maioria dos estudos sobre as florestas altomontanas restringiuse à análise da estrutura do componente arbóreo da formação, o que dificulta comparações e discussões mais aprofundadas com os resultados aqui apresentados. Em um dos poucos estudos no Brasil, que abordaram a florística de florestas altomontanas, Falkenberg (2003) listou 461 especies vegetais vasculares em florestas altomontanas com forte influência da Floresta Ombrófila Mista nos Aparados da Serra Geral em Santa Catarina e no Rio Grande do Sul, denominadas "matinhas nebulares". Cabe ressaltar que, neste trabalho, o autor incluiu espécies amostradas em áreas com interferência antrópica, como por exemplo, o pastejo bovino.

A família com maior riqueza específica foi Myrtaceae, com 34 espécies (10\% do total), seguida por Asteraceae (30;9\%), Orchidaceae (29; $8 \%)$, Rubiaceae $(17 ; 5 \%)$, Melastomataceae $(16 ; 5 \%)$, Poaceae $(12 ; 3 \%)$ e Bromeliaceae (11; 3\%) (Figura 3). Esses resultados são 

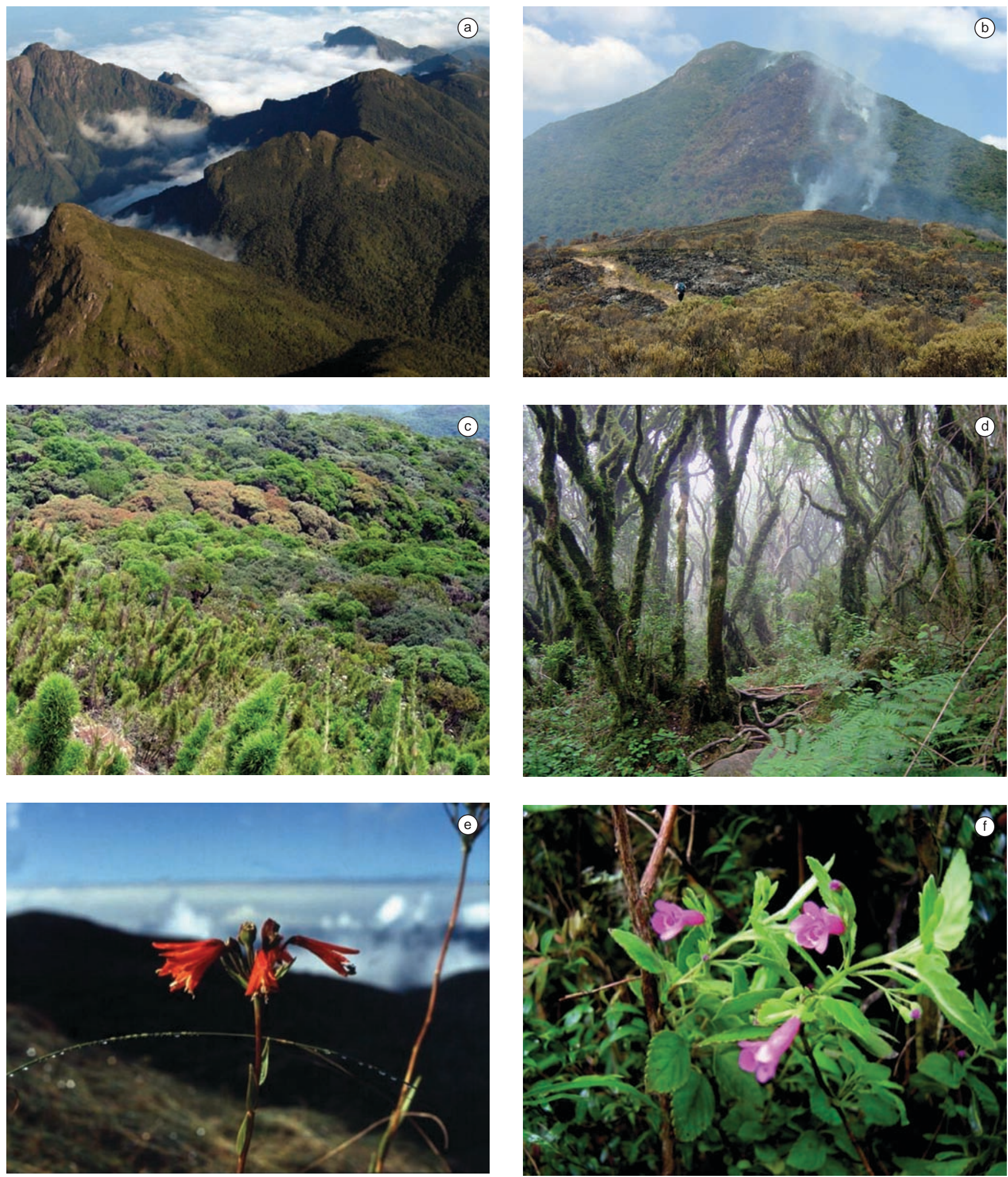

Figura 2. a) Florestas e campos altomontanos próximos aos cumes da Serra do Ibitiraquire no Paraná; b) Incêndio ocorrido em florestas primárias na encosta do Pico Caratuva, Serra do Ibitiraquire, em outubro de 2007; c) Aspecto do dossel da Floresta Ombrófila Densa Altomontana no Paraná; d) Aspecto do interior de Floresta Ombrófila Densa Altomontana típica no Pico Caratuva; e) Alstroemeria amabilis, uma das espécies descritas recentemente, típicas dos campos altomontanos e comum na borda da floresta altomontana; f) Espécie ainda não descrita de Hesperozigis (Élide dos Santos, comunicação pessoal).

Figure 2. a) Cloud Forests and grasslands near to the summits of the "Serra do Ibitiraquire" in the state of Paraná, Brazil; b) Fire in primary cloud forests on slope of Pico Caratuva, Serra do Ibitiraquire, October 2007; c) Aspect of the canopy of the studied cloud forests; d) Aspect of typical Upper Montane Cloud Forest in the Pico Caratuva; e) Alstroemeria amabilis, one of the species described recently, typical of the high altitude grasslands and common in the edge of cloud forests; f) Species still not described of the genus Hesperozigis (Élide dos Santos, personal communication). 
Tabela 2. Trabalhos utilizados para a comparação da florística arbórea através de análises de agrupamento e de índices de similaridade de Sörensen.

Table 2. Cloud forests sites used for arboreal floristic comparisons through Cluster analysis and Sörensen indexes.

$\begin{array}{ccc}\text { Complexo } & \text { Local } & \mathbf{N}^{\circ} \text { de espécies } \\ \text { arbóreas } & \text { Altitude } & \text { Referência } \\ & \text { envolvidas nas } & \\ & \text { análises do } & \\ & \text { presente trabalho } & \\ & & \end{array}$

\begin{tabular}{|c|c|c|c|c|}
\hline \multirow{4}{*}{$\begin{array}{l}\text { Serra da } \\
\text { Mantiqueira }\end{array}$} & Camanducaia, MG & 55 & 1.900 & França \& Stehmann (2004) \\
\hline & Parque Estadual Campos do Jordão, SP & 55 & $1.800-2.000$ & Robim et al. (1990) \\
\hline & Campos do Jordão, SP & 38 & 1.882 & Pereira-Silva et al. (2007) \\
\hline & Serra do Itatiaia, RJ & 30 & $>2.000$ & Brade (1956) \\
\hline \multirow{2}{*}{$\begin{array}{l}\text { Aparados } \\
\text { da Serra }\end{array}$} & Serra do Rio do Rastro, SC & 57 & $1.400^{*}$ & Falkenberg (2003) \\
\hline & Morro da Igreja, SC & 43 & $1.820^{*}$ & Falkenberg (2003) \\
\hline \multirow[t]{9}{*}{ Serra do Mar } & Serra da Baitaca, PR & 28 & $1.460^{*}$ & Roderjan (1994)/Portes et al. (2001) \\
\hline & Serra do Marumbi, PR & 35 & $1.545^{*} / 1.380^{*}$ & Rocha 1999/Koehler et al. (2002) \\
\hline & Serra do Araçatuba, PR & 17 & $1.610^{*}$ & Koehler et al. (2002) \\
\hline & Serra do Salto, PR & 20 & $1.390^{*}$ & Koehler et al. (2002) \\
\hline & Serra da Farinha Seca, PR & 20 & $1.457^{*}$ & Koehler et al. (2002) \\
\hline & Serra do Ibitiraquire, PR & 66 & $1.887^{*}$ & Presente trabalho \\
\hline & Serra da Igreja, PR & 56 & $1.376^{*}$ & Presente trabalho \\
\hline & Serra da Prata, PR & 49 & $1.502^{*}$ & Presente trabalho \\
\hline & Serra Gigante, PR & 60 & $1.069^{*}$ & Presente trabalho \\
\hline
\end{tabular}

*refere-se ao ponto culminante de cada serra/subserra

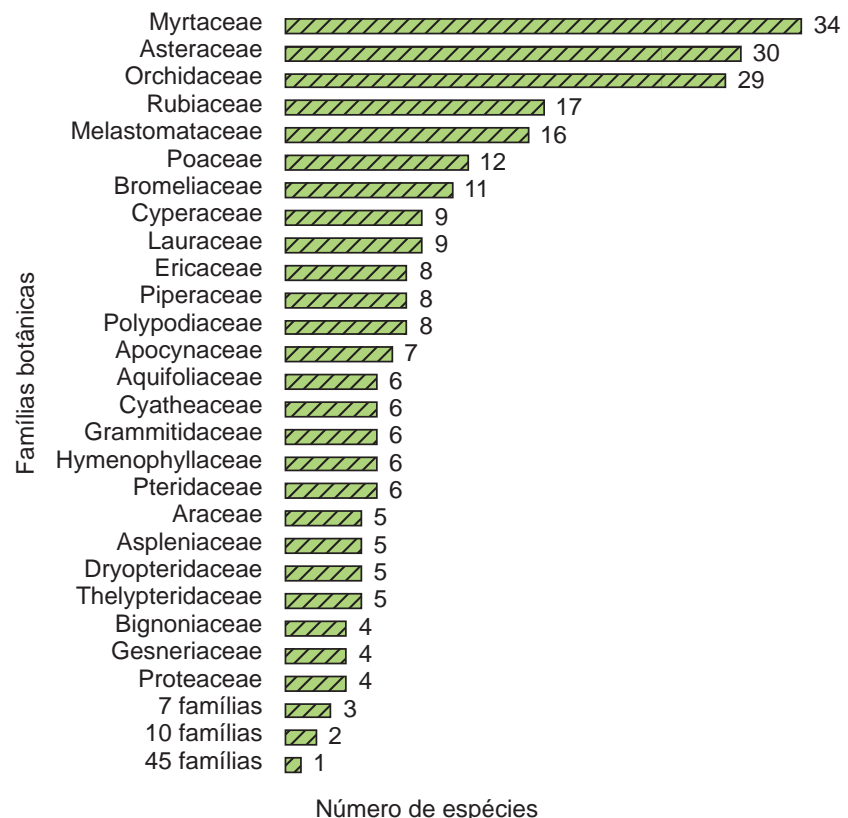

Figura 3. Número de espécies vasculares identificadas para cada família nas quatro serras amostradas no Paraná.

Figure 3. Number of vascular species for each family detected in the four cloud forests sampled in the present study, Southern Brazil. similares aos levantados por Falkenberg (2003) para as "matinhas nebulares" dos Aparados da Serra Geral, onde as famílias com maior número de espécies foram Asteraceae, Melastomataceae, Myrtaceae, Poaceae e Solanaceae, nesta ordem.

Os gêneros que apresentaram maior riqueza foram Myrceugenia (9 spp.); Leandra, Mikania e Baccharis (7 spp. cada) e Ilex, Eugenia e Peperomia (6 spp. cada). Novamente comparando os resultados com aqueles produzidos por Falkenberg (2003), Baccharis, Leandra, Solanum, Eupatorium, Myrceugenia e Mikania estão entre os gêneros mais importantes em número de espécies nas matinhas nebulares dos Aparados da Serra Geral. Percebe-se a coicidência de 4 gêneros importantes entre as duas áreas.

Do total de espécies encontradas, 231 (67\%) foram classificadas como típicas da formação (p.e. Ilex microdonta, Tabebuia catarinenisis e Myrceugenia franciscencis), 68 (19\%) como transicionais para florestas montanas (p.e. Aspidosperma pyricollum, Cabralea canjarana e Matayba guianensis) e 49 (14\%) como transicionais para campos de altitude (p.e. Alstroemeria amabilis, Hesperozigis rhododon e Gaylussacia brasiliensis). Espécies florestais típicas dos patamares montanos foram encontradas nos patamares altomontanos (encontrados somente indivíduos jovens) em montanhas com menores altitudes, como na Serra Gigante. Nestas áreas, as florestas altomontanas ocupam menores extensões e distribuem-se num gradiente altitudinal mais restrito. 
Tabela 3. Espécies vasculares da Floresta Ombrófila Densa Altomontana paranaense. Legenda: Forma biológica: ARB - arbusto; ARV - árvore; EPI - epífita; HER - herbácea; TRE - trepadeira; Local: IBI - Serra do Ibitiraquire; IGR - Serra da Igreja; PRA - Serra da Prata; GIG - Serra Gigante; Fitofisionomia: T - floresta altomontana típica; TC - transição para campo altomontano; TM - transição para floresta montana; Coletor: MBS - Maurício Bergamini Scheer; AYM - Alan Yukio Mocochinski; RTP - Ruddy Thomas Proença.

Table 3. List of cloud forest species in Sea Mountain Range in the state of Paraná, Southern Brazil. Legend: Life-form: ARB - shrub; ARV - tree; EPI - epiphyte; HER - herbaceous; TRE - climbers. Areas: IBI - Serra do Ibitiraquire; IGR - Serra da Igreja; PRA - Serra da Prata; GIG - Serra Gigante; Formation: T - typical upper montane cloud forest; TC - high altitude grassland transition; TM - lower montane transition; Collector: MBS - Maurício Bergamini Scheer; AYM - Alan Yukio Mocochinski; RTP - Ruddy Thomas Proença.

\begin{tabular}{|c|c|c|c|c|c|c|c|}
\hline \multirow[t]{2}{*}{ Família/Espécie } & \multirow[t]{2}{*}{ Forma } & \multicolumn{4}{|c|}{ Local } & \multirow[t]{2}{*}{ Fitofisionomia } & \multirow[t]{2}{*}{ Amostra } \\
\hline & & IBI & IGR & PRA & GIG & & \\
\hline \multicolumn{8}{|l|}{ ACANTHACEAE } \\
\hline Justicia cordifolia Heyne ex Wall. & ARB & $\mathrm{X}$ & & & & $\mathrm{T}$ & AYM 161 \\
\hline \multicolumn{8}{|l|}{ ALSTROEMERIACEAE } \\
\hline Alstroemeria amabilis M.C.Assis & HER & $\mathrm{X}$ & $\mathrm{X}$ & $\mathrm{X}$ & & $\mathrm{TC}$ & MBS 202 \\
\hline \multicolumn{8}{|l|}{ AMARYLLIDACEAE } \\
\hline Hippeastrum illustre (Vell.) Dutilh & HER & $\mathrm{X}$ & $\mathrm{X}$ & $\mathrm{X}$ & & $\mathrm{TC}$ & MBS 207 \\
\hline \multicolumn{8}{|l|}{ ANNONACEAE } \\
\hline Guatteria australis A.St.-Hil. & ARV & & & & $\mathrm{X}$ & TM & AYM 160 \\
\hline \multicolumn{8}{|l|}{ APOCYNACEAE } \\
\hline Aspidosperma pyricollum Müll. Arg. & ARV & & & & $\mathrm{X}$ & $\mathrm{TM}$ & AYM 159 \\
\hline Mandevilla atroviolacea (Stadelm.) Woodson & TREP & & & & $\mathrm{X}$ & TM & MBS 226 \\
\hline Mandevilla immaculata Woodson & TREP & $\mathrm{X}$ & $\mathrm{X}$ & & & $\mathrm{TM}$ & AYM 35 \\
\hline Orthosia dusenii (Malme) Fontella & HER & $\mathrm{X}$ & $\mathrm{X}$ & $\mathrm{X}$ & & $\mathrm{TC}$ & MBS 295 \\
\hline Oxypetalum sp. & TREP & & $\mathrm{X}$ & $\mathrm{X}$ & & $\mathrm{TC}$ & AYM 28 \\
\hline Indeterminada 1 & TREP & $X$ & & & & $\mathrm{~T}$ & AYM 311 \\
\hline Indeterminada 2 & TREP & & $\mathrm{X}$ & $\mathrm{X}$ & $\mathrm{X}$ & $\mathrm{T}$ & MBS 401 \\
\hline \multicolumn{8}{|l|}{ AQUIFOLIACEAE } \\
\hline Ilex dumosa Reissek & ARV & & & & $\mathrm{X}$ & TM & MBS s/n ${ }^{\circ}$ \\
\hline Ilex chamaedrifolia Reissek & ARV & $\mathrm{X}$ & $\mathrm{X}$ & $\mathrm{X}$ & $\mathrm{X}$ & $\mathrm{T}$ & AYM 157 \\
\hline Ilex microdonta Reissek & ARV & $\mathrm{X}$ & $\mathrm{X}$ & $\mathrm{X}$ & $\mathrm{X}$ & $\mathrm{T}$ & AYM 32 \\
\hline Ilex paraguariensis A.St.-Hil. & ARV & $\mathrm{X}$ & $\mathrm{X}$ & $\mathrm{X}$ & & $\mathrm{TM}$ & MBS 405 \\
\hline Ilex taubertiana Loes. & ARV & & & & $\mathrm{X}$ & TM & MBS 529 \\
\hline Ilex theezans Mart. & ARV & $X$ & & & $\mathrm{X}$ & $\mathrm{T}$ & MBS 528 \\
\hline \multicolumn{8}{|l|}{ ARACEAE } \\
\hline Anthurium acutum N.E.Brown & HER & & $\mathrm{X}$ & $\mathrm{X}$ & $\mathrm{X}$ & $\mathrm{T}$ & MBS 617 \\
\hline Anthurium cf. scandens (Aubl.) Engl. & HER & & $\mathrm{X}$ & & & $\mathrm{T}$ & MBS s/no \\
\hline Anthurium longicuspidatum Engl. & HER & & $\mathrm{X}$ & $\mathrm{X}$ & $\mathrm{X}$ & $\mathrm{T}$ & AYM 247 \\
\hline Philodendron cordatum Kunth. & HER & & & & $\mathrm{X}$ & $\mathrm{TM}$ & MBS 618 \\
\hline Philodendron sp. & HER & & $\mathrm{X}$ & & $\mathrm{X}$ & $\mathrm{T}$ & AYM 249 \\
\hline \multicolumn{8}{|l|}{ ARALIACEAE } \\
\hline Hydrocotyle quinqueloba Ruiz \& Pav. & HER & $\mathrm{X}$ & $\mathrm{X}$ & $\mathrm{X}$ & $\mathrm{X}$ & $\mathrm{T}$ & MBS 530 \\
\hline Hydrocotyle sp. & HER & $\mathrm{X}$ & & & & $\mathrm{T}$ & MBS s/n \\
\hline \multicolumn{8}{|l|}{ ARECACEAE } \\
\hline Geonoma schottiana Mart. & ARV & & $\mathrm{X}$ & & $\mathrm{X}$ & TM & AYM 7 \\
\hline \multicolumn{8}{|l|}{ ARISTOLOCHIACEAE } \\
\hline Aristolochia cf. triangularis Cham. \& Schl. & TREP & & & $\mathrm{X}$ & & TC & MBS 760 \\
\hline \multicolumn{8}{|l|}{ ASTERACEAE } \\
\hline $\begin{array}{l}\text { Austroeupatorium neglectum (B.L. Rob.) R.M.King \& } \\
\text { H.Rob. }\end{array}$ & $\mathrm{ARB}$ & $\mathrm{X}$ & & & $\mathrm{X}$ & TC & MBS 619 \\
\hline $\begin{array}{l}\text { Baccharis aracatubensis Teodoro \& Hatschbach ex } \\
\text { G.M.Barroso }\end{array}$ & $\mathrm{ARB}$ & & $\mathrm{X}$ & & & $\mathrm{TC}$ & MBS 625 \\
\hline Baccharis brachylaenoides DC. var brachylaenoides & ARV & $\mathrm{X}$ & $\mathrm{X}$ & $\mathrm{X}$ & $\mathrm{X}$ & $\mathrm{T}$ & AYM 3 \\
\hline Baccharis curitybensis Heering \& Dusén & $\mathrm{ARB}$ & $X$ & & $\mathrm{X}$ & & $\mathrm{TC}$ & AYM 129 \\
\hline Baccharis illinita DC. & $\mathrm{ARB}$ & & $\mathrm{X}$ & $\mathrm{X}$ & & $\mathrm{TC}$ & MBS 57 \\
\hline
\end{tabular}


Tabela 3. Continuação...

\begin{tabular}{|c|c|c|c|c|c|c|c|}
\hline \multirow[t]{2}{*}{ Família/Espécie } & \multirow[t]{2}{*}{ Forma } & \multicolumn{4}{|c|}{ Local } & \multirow[t]{2}{*}{ Fitofisionomia } & \multirow[t]{2}{*}{ Amostra } \\
\hline & & IBI & IGR & PRA & GIG & & \\
\hline Baccharis leucocephala Dusén & $\mathrm{ARB}$ & $\mathrm{X}$ & & & & $\mathrm{TC}$ & MBS 632 \\
\hline Baccharis tarchonanthoides Baker & $\mathrm{ARB}$ & $\mathrm{X}$ & & & & $\mathrm{TC}$ & AYM 257 \\
\hline Baccharis sp. & $\mathrm{ARB}$ & $\mathrm{X}$ & & & & TC & AYM 308 \\
\hline Critoniopsis quinqueflora (Less.) H.Rob. & $\mathrm{ARB}$ & $\mathrm{X}$ & $\mathrm{X}$ & $\mathrm{X}$ & & $\mathrm{T}$ & MBS 408 \\
\hline Dasyphyllum spinescens (Less.) Cabrera & $\mathrm{ARB}$ & $\mathrm{X}$ & & $\mathrm{X}$ & & $\mathrm{T}$ & AYM 259 \\
\hline Dasyphyllum sp. & $\mathrm{ARB}$ & $\mathrm{X}$ & & $\mathrm{X}$ & & $\mathrm{T}$ & MBS 700 \\
\hline Dendrophorbium limosus C. Jeffrey & TREP & $\mathrm{X}$ & $\mathrm{X}$ & $\mathrm{X}$ & $\mathrm{X}$ & $\mathrm{TC}$ & MBS 13 \\
\hline Grazielia cf. serrata (Spreng.) R.M.King \& H.Rob. & HER & $\mathrm{X}$ & $\mathrm{X}$ & & & $\mathrm{TC}$ & AYM 256 \\
\hline Heterocondylus alatus (Vell.) R.M.King \& H.Rob. & $\mathrm{ARB}$ & $\mathrm{X}$ & $X$ & $\mathrm{X}$ & & $\mathrm{T}$ & MBS 694 \\
\hline Mikania campanulata Gardner & TREP & $X$ & & $\mathrm{X}$ & $\mathrm{X}$ & $\mathrm{T}$ & MBS 634 \\
\hline Mikania involucrata Hook. \& Arn. & HER & & & & $\mathrm{X}$ & TC & AYM258 \\
\hline Mikania lanuginosa DC. & HER & $\mathrm{X}$ & $\mathrm{X}$ & & $\mathrm{X}$ & $\mathrm{T}$ & MBS 621 \\
\hline Mikania lindbergii Baker & TREP & & & & $\mathrm{X}$ & $\mathrm{TM}$ & MBS 635 \\
\hline Mikania paranaensis Dusén & TREP & $\mathrm{X}$ & & & & $\mathrm{TC}$ & MBS s/n ${ }^{\circ}$ \\
\hline Mikania sp. 1 & TREP & & & $\mathrm{X}$ & & TC & AYM139 \\
\hline Mikania sp. 2 & TREP & & $\mathrm{X}$ & & & $\mathrm{TC}$ & MBS s/n ${ }^{\circ}$ \\
\hline Pentacalia desiderabilis (Vell.) Cuatrec. & TREP & $\mathrm{X}$ & $\mathrm{X}$ & $\mathrm{X}$ & & $\mathrm{T}$ & AYM 250 \\
\hline Piptocarpha densifolia Dusén ex. G.L.Smith & ARV & $\mathrm{X}$ & $\mathrm{X}$ & & $\mathrm{X}$ & $\mathrm{T}$ & MBS 627 \\
\hline Symphyopappus lymansmithii B.L.Rob. & ARV & $\mathrm{X}$ & $\mathrm{X}$ & & & $\mathrm{T}$ & MBS 624 \\
\hline Trixis brasiliensis (L.) DC. & HER & $\mathrm{X}$ & $\mathrm{X}$ & $\mathrm{X}$ & & $\mathrm{T}$ & AYM 252 \\
\hline Verbesina glabrata Hook \& Arn. & HER & & $\mathrm{X}$ & $\mathrm{X}$ & & $\mathrm{T}$ & MBS 411 \\
\hline Indeterminada 1 & ARB & & $\mathrm{X}$ & & & $\mathrm{T}$ & MBS 407 \\
\hline Indeterminada 2 & $\mathrm{ARB}$ & & $\mathrm{X}$ & & & $\mathrm{T}$ & MBS 434 \\
\hline Indeterminada 3 & $\mathrm{ARB}$ & & $\mathrm{X}$ & & & $\mathrm{T}$ & MBS 409 \\
\hline Indeterminada 4 & $\mathrm{ARB}$ & & $X$ & & & $\mathrm{~T}$ & MBS 410 \\
\hline \multicolumn{8}{|l|}{ BEGONIACEAE } \\
\hline Begonia aff. angulata Vell. & HER & $\mathrm{X}$ & $\mathrm{X}$ & $X$ & & $\mathrm{~T}$ & AYM 163 \\
\hline \multicolumn{8}{|l|}{ BERBERIDACEAE } \\
\hline Berberis laurina Billb. & $\mathrm{ARB}$ & $\mathrm{X}$ & & & & $\mathrm{TC}$ & MBS 121 \\
\hline \multicolumn{8}{|l|}{ BIGNONIACEAE } \\
\hline Anemopaegma chamberlaynii (Sims) Bureau \& K.Schum. & TREP & $\mathrm{X}$ & $\mathrm{X}$ & $\mathrm{X}$ & & $\mathrm{T}$ & MBS 508 \\
\hline Anemopaegma prostratum DC. & TREP & & & & $\mathrm{X}$ & $\mathrm{T}$ & MBS 535 \\
\hline Anemopaegma sp. & TREP & & & & & $\mathrm{TC}$ & AYM 373 \\
\hline Tabebuia catarinensis A. Gentry & ARV & $\mathrm{X}$ & $\mathrm{X}$ & $\mathrm{X}$ & $\mathrm{X}$ & $\mathrm{T}$ & MBS 10 \\
\hline \multicolumn{8}{|l|}{ BROMELIACEAE } \\
\hline Aechmea cf. fasciata (Lindl.) Baker & HER & & & & $\mathrm{X}$ & TM & AYM 374 \\
\hline Aechmea cylindrata Lindm. & EPI & & $\mathrm{X}$ & $\mathrm{X}$ & & $\mathrm{TM}$ & $\operatorname{MBS~s} / \mathrm{n}^{\circ}$ \\
\hline Aechmea ornata Baker & HER & $\mathrm{X}$ & $X$ & $\mathrm{X}$ & & $\mathrm{T}$ & $\operatorname{MBS~s} / \mathrm{n}^{\circ}$ \\
\hline Aechmea ornata Baker var. hoeneana L.B.Sm. & HER & & & & $\mathrm{X}$ & $\mathrm{T}$ & MBS 538 \\
\hline Nidularium campo-alegrensis Leme & HER & & $\mathrm{X}$ & $X$ & $\mathrm{X}$ & $\mathrm{T}$ & MBS 748 \\
\hline Pitcairnia flammea Lindl. var floccosa L.B.Sm. & HER & $\mathrm{X}$ & & $\mathrm{X}$ & $\mathrm{X}$ & $\mathrm{TM}$ & MBS $s / n^{\circ}$ \\
\hline Vriesea altodaserrae L.B. Sm. & HER & & $X$ & & $\mathrm{X}$ & $\mathrm{T}$ & AYM 167 \\
\hline Vriesea heterostachys (Baker) L.B.Sm. & HER & & & & $\mathrm{X}$ & $\mathrm{TM}$ & AYM 366 \\
\hline Vriesea guttata Linden \& André & HER & & & $\mathrm{X}$ & $\mathrm{X}$ & TM & MBS 537 \\
\hline Vriesea platynema var. variegata Gaudich. & HER & $\mathrm{X}$ & $\mathrm{X}$ & $\mathrm{X}$ & $X$ & $\mathrm{TC}$ & AYM 166 \\
\hline Wittrockia cyathiformis (Vellozo) Leme & HER & & $\mathrm{X}$ & & $\mathrm{X}$ & $\mathrm{T}$ & MBS 416 \\
\hline \multicolumn{8}{|l|}{ CACTACEAE } \\
\hline Hatiora gaertneri (Regel) Barthlott & EPI & $\mathrm{X}$ & & & & $\mathrm{T}$ & MBS 539 \\
\hline Hatiora rosea (Lagerh.) Barthlott & EPI & & & $\mathrm{X}$ & & $\mathrm{T}$ & MBS 507 \\
\hline Rhipsalis sp. & EPI & $\mathrm{X}$ & & & & $\mathrm{T}$ & AYM 98 \\
\hline
\end{tabular}


Tabela 3. Continuação...

\begin{tabular}{|c|c|c|c|c|c|c|c|}
\hline \multirow[t]{2}{*}{ Família/Espécie } & \multirow[t]{2}{*}{ Forma } & \multicolumn{4}{|c|}{ Local } & \multirow[t]{2}{*}{ Fitofisionomia } & \multirow[t]{2}{*}{ Amostra } \\
\hline & & IBI & IGR & PRA & GIG & & \\
\hline \multicolumn{8}{|l|}{ CAMPANULACEAE } \\
\hline Siphocampylus eichleri Kanitz & HER & $\mathrm{X}$ & & & & $\mathrm{TC}$ & AYM 169 \\
\hline Siphocampylus fimbriatus Regel & HER & $\mathrm{X}$ & $\mathrm{X}$ & & $\mathrm{X}$ & $\mathrm{T}$ & MBS 234 \\
\hline Siphocampylus fulgens Leb. & HER & & $\mathrm{X}$ & $\mathrm{X}$ & $\mathrm{X}$ & $\mathrm{T}$ & AYM 45 \\
\hline \multicolumn{8}{|l|}{ CARDIOPTERIDACEAE } \\
\hline Citronella paniculata (Mart.) Howard & ARV & $\mathrm{X}$ & $X$ & $\mathrm{X}$ & $\mathrm{X}$ & $\mathrm{T}$ & AYM 182 \\
\hline \multicolumn{8}{|l|}{ CELASTRACEAE } \\
\hline Maytenus glaucescens Reiss. & ARV & $\mathrm{X}$ & $\mathrm{X}$ & $\mathrm{X}$ & & $\mathrm{T}$ & MBS 418 \\
\hline Maytenus urbaniana Loes. & ARV & $\mathrm{X}$ & & $\mathrm{X}$ & & $\mathrm{T}$ & MBS 541 \\
\hline \multicolumn{8}{|l|}{ CHLORANTACEAE } \\
\hline Hedyosmum brasiliense Miq. & $\mathrm{ARB}$ & & & & $\mathrm{X}$ & TM & MBS 544 \\
\hline \multicolumn{8}{|l|}{ CLETHRACEAE } \\
\hline Clethra scabra Sleumer var. variegata (Meissner) Sleumer & ARV & & & & $\mathrm{X}$ & $\mathrm{T}$ & AYM 172 \\
\hline Clethra uleana Sleumer & ARV & $\mathrm{X}$ & $X$ & $\mathrm{X}$ & & $\mathrm{T}$ & MBS 353 \\
\hline \multicolumn{8}{|l|}{ CORNACEAE } \\
\hline Griselinia ruscifolia (Clos.) Taub. & TREP & $\mathrm{X}$ & $\mathrm{X}$ & $\mathrm{X}$ & $\mathrm{X}$ & $\mathrm{T}$ & AYM 46 \\
\hline \multicolumn{8}{|l|}{ CUCURBITACEAE } \\
\hline Cayaponia cf. palinata Cogn. & TREP & $\mathrm{X}$ & $\mathrm{X}$ & & & $\mathrm{T}$ & AYM 318 \\
\hline \multicolumn{8}{|l|}{ CUNONIACEAE } \\
\hline Weinmannia humilis Engler & ARV & $\mathrm{X}$ & $X$ & $\mathrm{X}$ & & $\mathrm{T}$ & MBS 16 \\
\hline Weinmannia paullinifolia Pohl ex Ser. & ARV & & $\mathrm{X}$ & & $\mathrm{X}$ & $\mathrm{TM}$ & AYM 173 \\
\hline \multicolumn{8}{|l|}{ CYPERACEAE } \\
\hline Carex sp. & HER & & $X$ & & & $\mathrm{TC}$ & AYM 103 \\
\hline Pleurostachys beyrichii (Nees) Steud. & HER & $\mathrm{X}$ & $\mathrm{X}$ & $\mathrm{X}$ & $\mathrm{X}$ & $\mathrm{T}$ & MBS 419 \\
\hline Rhynchospora cf. exaltata Kunth & HER & $\mathrm{X}$ & $\mathrm{X}$ & $\mathrm{X}$ & $\mathrm{X}$ & $\mathrm{TC}$ & MBS 468 \\
\hline Rhynchospora cf. splendens Lindm. & HER & $\mathrm{X}$ & $\mathrm{X}$ & $\mathrm{X}$ & & $\mathrm{T}$ & MBS 419 \\
\hline Rhynchospora sp. & HER & $\mathrm{X}$ & & & & $\mathrm{T}$ & MBS 684 \\
\hline Scleria panicoides Kunth & HER & & & $\mathrm{X}$ & $\mathrm{X}$ & $\mathrm{T}$ & AYM 298 \\
\hline Indeterminada 1 & HER & & $\mathrm{X}$ & & & $\mathrm{T}$ & AYM 47 \\
\hline Indeterminada 2 & HER & & $\mathrm{X}$ & & & $\mathrm{T}$ & AYM 104 \\
\hline Indeterminada 3 & HER & & $\mathrm{X}$ & & & $\mathrm{T}$ & AYM 103 \\
\hline \multicolumn{8}{|l|}{ DIOSCOREACEAE } \\
\hline Dioscorea sanpaulensis $\mathrm{R}$. Knuth & TREP & $\mathrm{X}$ & $\mathrm{X}$ & & $\mathrm{X}$ & $\mathrm{TC}$ & AYM 174 \\
\hline \multicolumn{8}{|l|}{ ERICACEAE } \\
\hline $\begin{array}{l}\text { Agarista niederleinii (Sleumer) Judd var. acutifolia W.S. } \\
\text { Judd. }\end{array}$ & ARV & $\mathrm{X}$ & & $\mathrm{X}$ & & $\mathrm{TC}$ & MBS 421 \\
\hline Agarista niederleinii (Sleumer) Judd var. niederleinii & ARV & & $\mathrm{X}$ & & & $\mathrm{TC}$ & MBS 549 \\
\hline Agarista sp. & ARV & & & & $\mathrm{X}$ & $\mathrm{TC}$ & AYM 376 \\
\hline $\begin{array}{l}\text { Gaultheria serrata (Vell.) Sleum. ex Kin.-Gouv. var. } \\
\text { organensis (Meisn.) Luteyn }\end{array}$ & $\mathrm{ARB}$ & $\mathrm{X}$ & & & & $\mathrm{TC}$ & MBS s/n ${ }^{\circ}$ \\
\hline Gaultheria sp. & $\mathrm{ARB}$ & $\mathrm{X}$ & & & & $\mathrm{TC}$ & MBS 395 \\
\hline Gaylussacia brasiliensis (Spr.) Meissn. var. brasiliensis & $\mathrm{ARB}$ & $\mathrm{X}$ & $\mathrm{X}$ & $\mathrm{X}$ & $\mathrm{X}$ & $\mathrm{TC}$ & MBS 378 \\
\hline Gaylussacia caratuvensis R.R.Silva \& Cervi & $\mathrm{ARB}$ & $\mathrm{X}$ & & & & $\mathrm{TC}$ & MBS 77 \\
\hline Gaylussacia sp. & $\mathrm{ARB}$ & & $\mathrm{X}$ & & & $\mathrm{TC}$ & MBS 394 \\
\hline \multicolumn{8}{|l|}{ ERYTHROXYLACEAE } \\
\hline Erythroxylum gonoclados (Mart.) O.E. Schulz & ARB & $\mathrm{X}$ & $\mathrm{X}$ & $\mathrm{X}$ & & $\mathrm{T}$ & MBS 550 \\
\hline \multicolumn{8}{|l|}{ ESCALLONIACEAE } \\
\hline Escallonia laevis (Vell.) Sleum. & ARV & $\mathrm{X}$ & & & & $\mathrm{TC}$ & MBS 247 \\
\hline \multicolumn{8}{|l|}{ EUPHORBIACEAE } \\
\hline Alchornea triplinervia (Spreng.) Müll. Arg. & ARV & $\mathrm{X}$ & $\mathrm{X}$ & & & $\mathrm{TM}$ & $\operatorname{MBS~s} / \mathrm{n}^{\circ}$ \\
\hline Phyllanthus carolinensis Walter & $\mathrm{ARB}$ & & $\mathrm{X}$ & & & $\mathrm{T}$ & MBS 397 \\
\hline
\end{tabular}


Tabela 3. Continuação...

\begin{tabular}{|c|c|c|c|c|c|c|c|}
\hline \multirow[t]{2}{*}{ Família/Espécie } & \multirow[t]{2}{*}{ Forma } & \multicolumn{4}{|c|}{ Local } & \multirow[t]{2}{*}{ Fitofisionomia } & \multirow[t]{2}{*}{ Amostra } \\
\hline & & IBI & IGR & PRA & GIG & & \\
\hline \multicolumn{8}{|l|}{ FABACEAE - CAESALPINIOIDEAE } \\
\hline $\begin{array}{l}\text { Senna organensis (Glaz. ex Harms) Irw. \& Barn var. } \\
\text { extratropica Irwin \& Barneby }\end{array}$ & ARV & $\mathrm{X}$ & & & $X$ & TC & AYM 223 \\
\hline \multicolumn{8}{|l|}{ FABACEAE - MIMOSOIDEAE } \\
\hline Inga barbata Bentham & ARV & $\mathrm{X}$ & $\mathrm{X}$ & & $\mathrm{X}$ & $\mathrm{T}$ & MBS 592 \\
\hline \multicolumn{8}{|l|}{ GENTIANACEAE } \\
\hline Macrocarpaea rubra Malme & HER & & $\mathrm{X}$ & $\mathrm{X}$ & $X$ & $\mathrm{~T}$ & MBS 115 \\
\hline \multicolumn{8}{|l|}{ GESNERIACEAE } \\
\hline Nematanthus australis Chautems & EPI & $\mathrm{X}$ & $\mathrm{X}$ & $\mathrm{X}$ & $X$ & $\mathrm{~T}$ & MBS s/no \\
\hline Nematanthus cf. tessmanii (Hoehne) Chautems & EPI & & $\mathrm{X}$ & & & $\mathrm{TM}$ & MBS 422 \\
\hline Sinningia aff. magnifica (Otto \& A.Dietr.) Wiehle & HER & $\mathrm{X}$ & & & & $\mathrm{TC}$ & AYM 369 \\
\hline Sinningia mauroana Chateums & EPI & & & & $\mathrm{X}$ & $\mathrm{T}$ & MBS 274 \\
\hline \multicolumn{8}{|l|}{ IRIDACEAE } \\
\hline Sisyrinchium vaginatum Spreng. & HER & & & $\mathrm{X}$ & & $\mathrm{TC}$ & MBS 520 \\
\hline \multicolumn{8}{|l|}{ LAMIACEAE } \\
\hline Hesperozigis rhododon Epling & $\mathrm{ARB}$ & $\mathrm{X}$ & $\mathrm{X}$ & $\mathrm{X}$ & & $\mathrm{TC}$ & MBS s/no \\
\hline Hesperozigis sp. inedt. & HER & $\mathrm{X}$ & & & & $\mathrm{TC}$ & MBS $s / n^{\circ}$ \\
\hline Salvia melissiflora Bentham & HER & $\mathrm{X}$ & & & & $\mathrm{T}$ & MBS $s / n^{\circ}$ \\
\hline \multicolumn{8}{|l|}{ LAURACEAE } \\
\hline Ocotea porosa (Nees \& C. Mart.) Barroso & ARV & $\mathrm{X}$ & $\mathrm{X}$ & $\mathrm{X}$ & $\mathrm{X}$ & $\mathrm{T}$ & AYM 214 \\
\hline Ocotea vaccinioides (Meisn.) Mez & ARV & & $\mathrm{X}$ & & $\mathrm{X}$ & $\mathrm{T}$ & MBS 504 \\
\hline Ocotea pulchella Mart. & ARV & & $\mathrm{X}$ & & & $\mathrm{TM}$ & MBS 426 \\
\hline Ocotea bicolor Vattimo & ARV & $X$ & & & $\mathrm{X}$ & $\mathrm{T}$ & AYM 215 \\
\hline Ocotea tristis Mart. ex Nees & ARV & & & & $\mathrm{X}$ & $\mathrm{T}$ & MBS 494 \\
\hline Persea alba Nees & ARV & $\mathrm{X}$ & & & $\mathrm{X}$ & $\mathrm{T}$ & AYM 213 \\
\hline Persea pyrifolia (Ness.) Kopp. & ARV & $\mathrm{X}$ & $\mathrm{X}$ & $\mathrm{X}$ & & $\mathrm{T}$ & AYM 57 \\
\hline Nectandra cf. membranacea (Sw.) Griseb. & ARV & $X$ & & & & $\mathrm{~T}$ & MBS 750 \\
\hline Cinamomum cf. hatschbachii Vattimo & ARV & & & $\mathrm{X}$ & $\mathrm{X}$ & $\mathrm{TM}$ & MBS s/no \\
\hline \multicolumn{8}{|l|}{ LENTIBULARIACEAE } \\
\hline Utricularia reniformis A. St. Hill. & HER & $\mathrm{X}$ & $\mathrm{X}$ & $X$ & & $\mathrm{~T}$ & AYM 217 \\
\hline \multicolumn{8}{|l|}{ LOGANIACEAE } \\
\hline Spigelia tetraptera Taub. ex Glaz. & $\mathrm{ARB}$ & $\mathrm{X}$ & & $\mathrm{X}$ & & $\mathrm{T}$ & AYM 138 \\
\hline \multicolumn{8}{|l|}{ LORANTHACEAE } \\
\hline Struthanthus cf. vulgaris Mart. & TREP & & & & $\mathrm{X}$ & $\mathrm{T}$ & MBS 106 \\
\hline Struthanthus complexus Eichler & TREP & $\mathrm{X}$ & & & & $\mathrm{T}$ & MBS 685 \\
\hline Struthanthus sp. & TREP & $X$ & & & & $\mathrm{~T}$ & AYM 309 \\
\hline \multicolumn{8}{|l|}{ MALPIGHIACEAE } \\
\hline Heteropterys nitida H.B. \& K. & TREP & & & & $X$ & $\mathrm{TM}$ & MBS 323 \\
\hline \multicolumn{8}{|l|}{ MELASTOMATACEAE } \\
\hline Leandra acutiflora (Naudin) Cogn. & $\mathrm{ARB}$ & & $\mathrm{X}$ & & & $\mathrm{T}$ & MBS 429 \\
\hline Leandra carassana (DC.) Cogn. & $\mathrm{ARB}$ & & & $\mathrm{X}$ & & $\mathrm{T}$ & MBS 509 \\
\hline Leandra hatschbachii Brade & $\mathrm{ARB}$ & $\mathrm{X}$ & $\mathrm{X}$ & & & $\mathrm{T}$ & MBS 656 \\
\hline Leandra $\mathrm{cf}$. multiplinervis (Naudin) Cogn. & $\mathrm{ARB}$ & $\mathrm{X}$ & & & & $\mathrm{T}$ & MBS 655 \\
\hline Leandra cf. quinquedentata (Mart.\& Schrank) Cogn. & $\mathrm{ARB}$ & $\mathrm{X}$ & $\mathrm{X}$ & $\mathrm{X}$ & $\mathrm{X}$ & $\mathrm{T}$ & MBS 239 \\
\hline Leandra sp. 1 & $\mathrm{ARB}$ & $\mathrm{X}$ & & & & $\mathrm{T}$ & AYM 321 \\
\hline Leandra sp. 2 & HER & & & $\mathrm{X}$ & & $\mathrm{T}$ & MBS 509 \\
\hline Miconia cubatanensis Hoehne & $\mathrm{ARB}$ & & $\mathrm{X}$ & $\mathrm{X}$ & & $\mathrm{TM}$ & AYM 276 \\
\hline Miconia lymanii Wurdack & $\mathrm{ARB}$ & $\mathrm{X}$ & $\mathrm{X}$ & $\mathrm{X}$ & $\mathrm{X}$ & $\mathrm{T}$ & AYM 62 \\
\hline Miconia ramboi Brade & ARV & $\mathrm{X}$ & & & & & MBS $s / n^{\circ}$ \\
\hline Miconia sp. & $\mathrm{ARB}$ & & $\mathrm{X}$ & & & $\mathrm{T}$ & AYM 279 \\
\hline
\end{tabular}


Tabela 3. Continuação...

\begin{tabular}{|c|c|c|c|c|c|c|c|}
\hline \multirow[t]{2}{*}{ Família/Espécie } & \multirow[t]{2}{*}{ Forma } & \multicolumn{4}{|c|}{ Local } & \multirow[t]{2}{*}{ Fitofisionomia } & \multirow[t]{2}{*}{ Amostra } \\
\hline & & IBI & IGR & PRA & GIG & & \\
\hline Tibouchina hospita (DC) Cogn. & ARB & & & & & $\mathrm{TC}$ & MBS 661 \\
\hline Tibouchina hatschbachii Wurdack & ARB & $\mathrm{X}$ & & & $\mathrm{X}$ & $\mathrm{TC}$ & MBS 325 \\
\hline Tibouchina reitzii Brade & ARV & $\mathrm{X}$ & $\mathrm{X}$ & $\mathrm{X}$ & $X$ & $\mathrm{~T}$ & MBS 286 \\
\hline Indeterminada 1 & ARB & $\mathrm{X}$ & & & & $\mathrm{T}$ & AYM 278 \\
\hline Indeterminada 2 & $\mathrm{ARB}$ & $\mathrm{X}$ & & & & $\mathrm{T}$ & MBS 696 \\
\hline \multicolumn{8}{|l|}{ MELIACEAE } \\
\hline Cabralea canjerana (Vell.) Mart. & ARV & & $\mathrm{X}$ & $\mathrm{X}$ & $\mathrm{X}$ & TM & $\operatorname{MBS} \mathrm{s} / \mathrm{n}^{\circ}$ \\
\hline \multicolumn{8}{|l|}{ MONIMIACEAE } \\
\hline Mollinedia cf. uleana Perk. & ARV & $\mathrm{X}$ & $\mathrm{X}$ & $\mathrm{X}$ & & $\mathrm{T}$ & AYM 141 \\
\hline \multicolumn{8}{|l|}{ MYRSINACEAE } \\
\hline Conomorpha peruviana A.DC. & ARV & & $\mathrm{X}$ & & $\mathrm{X}$ & $\mathrm{TM}$ & MBS 596 \\
\hline Myrsine altomontana M.F.Freitas \& Kin.-Gouv. & ARV & $\mathrm{X}$ & $\mathrm{X}$ & $\mathrm{X}$ & $\mathrm{X}$ & $\mathrm{T}$ & MBS 81 \\
\hline Myrsine cf. umbellata Mart. ex DC. & ARV & $\mathrm{X}$ & $\mathrm{X}$ & & $\mathrm{X}$ & $\mathrm{TM}$ & AYM 225 \\
\hline \multicolumn{8}{|l|}{ MYRTACEAE } \\
\hline Blepharocalyx salicifolius (H.B.K.) O.Berg & ARV & $\mathrm{X}$ & $\mathrm{X}$ & $\mathrm{X}$ & $\mathrm{X}$ & $\mathrm{T}$ & AYM 146 \\
\hline Calyptranthes obovata Kiaersk. & ARV & $\mathrm{X}$ & & & & $\mathrm{T}$ & MBS 640 \\
\hline Calyptranthes sp. & ARV & $\mathrm{X}$ & & & & $\mathrm{T}$ & AYM 285 \\
\hline Eugenia cf. oeidocarpa O.Berg. & ARV & & & & $X$ & $\mathrm{TM}$ & AYM 293 \\
\hline Eugenia eurysepala Kiaersk. & ARV & & & $\mathrm{X}$ & $\mathrm{X}$ & $\mathrm{TM}$ & MBS 665 \\
\hline Eugenia handroana D.Legrand & ARV & $\mathrm{X}$ & & & $\mathrm{X}$ & $\mathrm{TM}$ & AYM 133 \\
\hline Eugenia neomyrtifolia Sobral & ARB & $\mathrm{X}$ & & $\mathrm{X}$ & & $\mathrm{T}$ & AYM 126 \\
\hline Eugenia sclerocalyx D.Legrand & ARV & $\mathrm{X}$ & $\mathrm{X}$ & $\mathrm{X}$ & & $\mathrm{T}$ & AYM 68 \\
\hline Eugenia sp. & ARV & $\mathrm{X}$ & $\mathrm{X}$ & & & $\mathrm{T}$ & AYM 288 \\
\hline Gomidesia sellowiana Berg. & ARV & $\mathrm{X}$ & $\mathrm{X}$ & $\mathrm{X}$ & $\mathrm{X}$ & $\mathrm{T}$ & AYM 320 \\
\hline Myrceugenia alpigena (DC.) Landrum & ARV & $\mathrm{X}$ & & & & $\mathrm{T}$ & AYM 264 \\
\hline Myrceugenia euosma (O.Berg) D.Legrand & ARV & $\mathrm{X}$ & $\mathrm{X}$ & $\mathrm{X}$ & $\mathrm{X}$ & $\mathrm{T}$ & MBS 501 \\
\hline Myrceugenia franciscensis (O.Berg) Landrum & ARV & $X$ & $\mathrm{X}$ & $\mathrm{X}$ & $\mathrm{X}$ & $\mathrm{T}$ & AYM 130 \\
\hline Myrceugenia myrcioides (Cambess.) O.Berg. & ARV & $\mathrm{X}$ & & & & $\mathrm{T}$ & MBS 687 \\
\hline Myrceugenia ovata (Hook \& Arn.) O.Berg. & ARV & $\mathrm{X}$ & $\mathrm{X}$ & $\mathrm{X}$ & & $\mathrm{T}$ & MBS 649 \\
\hline Myrceugenia pilotantha (Kiaersk.) Landrum & ARV & $\mathrm{X}$ & & $\mathrm{X}$ & & $\mathrm{T}$ & MBS 662 \\
\hline Myrceugenia seriatoramosa (Kiaersk.) D.Legrand \& Krausel & ARV & $\mathrm{X}$ & $\mathrm{X}$ & $\mathrm{X}$ & & $\mathrm{T}$ & AYM 291 \\
\hline Myrceugenia sp. 1 & ARV & $\mathrm{X}$ & $\mathrm{X}$ & $\mathrm{X}$ & & $\mathrm{T}$ & MBS 692 \\
\hline Myrceugenia sp. 2 & ARV & & & $\mathrm{X}$ & & $\mathrm{T}$ & MBS 679 \\
\hline Myrcia breviramis (Berg.) D.Legrand & ARV & $\mathrm{X}$ & $\mathrm{X}$ & $\mathrm{X}$ & $\mathrm{X}$ & $\mathrm{T}$ & MBS 65 \\
\hline Myrcia cf. dicrophylla D.Legrand & ARV & & & & $\mathrm{X}$ & $\mathrm{TM}$ & MBS 503 \\
\hline Myrcia cf. freyreissiana (O. Berg.) Kiaersk. & ARV & & & & $X$ & $\mathrm{TM}$ & MBS 669 \\
\hline Myrcia cf. rostrata DC. & ARV & & $\mathrm{X}$ & & & $\mathrm{TM}$ & AYM 268 \\
\hline Myrcia obtecta Kiaersk. & ARV & $\mathrm{X}$ & & $\mathrm{X}$ & $\mathrm{X}$ & $\mathrm{T}$ & MBS 641 \\
\hline Myrcia richardiana (O.Berg) Kiaersk. & ARV & $\mathrm{X}$ & $\mathrm{X}$ & $\mathrm{X}$ & $X$ & $\mathrm{~T}$ & MBS 496 \\
\hline Pimenta pseudocaryophyllus (Gomes) Landrum & ARV & $\mathrm{X}$ & $\mathrm{X}$ & $\mathrm{X}$ & $X$ & $\mathrm{~T}$ & AYM 270 \\
\hline Plinia cordifolia (D.Legrand.) Sobral & ARV & $\mathrm{X}$ & $\mathrm{X}$ & $\mathrm{X}$ & & $\mathrm{T}$ & MBS 365 \\
\hline Psidium sp. & ARV & & & & $X$ & $\mathrm{TM}$ & AYM 289 \\
\hline Siphoneugena reitzii D.Legrand & ARV & $\mathrm{X}$ & $\mathrm{X}$ & $\mathrm{X}$ & $\mathrm{X}$ & $\mathrm{T}$ & AYM 123 \\
\hline Indeterminada 1 & ARV & & $\mathrm{X}$ & & & $\mathrm{T}$ & $\operatorname{MBS} \mathrm{s} / \mathrm{n}^{\circ}$ \\
\hline Indeterminada 2 & ARV & & & & $X$ & $\mathrm{~T}$ & AYM 288 \\
\hline Indeterminada 3 & ARV & $\mathrm{X}$ & & $\mathrm{X}$ & & $\mathrm{T}$ & MBS 515 \\
\hline Indeterminada 4 & ARV & & & $\mathrm{X}$ & & $\mathrm{T}$ & AYM 145 \\
\hline Indeterminada 5 & ARV & $\mathrm{X}$ & & & & $\mathrm{T}$ & MBS 687 \\
\hline \multicolumn{8}{|l|}{ NYCTAGINACEAE } \\
\hline Guapira opposita (Vell.) Reitz & ARV & & & & $X$ & $\mathrm{TM}$ & MBS 232 \\
\hline
\end{tabular}


Tabela 3. Continuação...

\section{Família/Espécie}

Forma

\section{IBI}

OCHNACEAE

Ouratea vaccinioides Engl.

ONAGRACEAE

Fuchsia regia (Vand. ex. Vell) Muniz var. serrae P.E.Berry ORCHIDACEAE

Bulbophyllum napellii Lindl.

Dichaea anchorifera Cogn.

Encyclia cf. patens Hook.

Encyclia fausta (Rchb. f. ex Cogn.) Pabst

Epidendron ellipticum Grah.

Gomesa sp.

Maxillaria bradei Schltr. ex Hoehne

Maxillaria picta Hook.

Maxillaria sp.

Octomeria cf. iguapensis Schltr.

Octomeria cf. robusta Barb.Rodr.

Oncidium flexuosum (Kunth) Lindl.

Oncidium sp.

Phymatidium tillandsioides Barb.Rodr.

Pleurothallis sp. 1

Pleurothallis sp. 2

Pleurothallis sp. 3

Prescottia cf. stachyoides (Sw.) Lindl.

Promenaea xanthina Lindl.

Promenaea sp.

Sauroglossum sp.

Scaphyglottis modesta (Rchb. f.) Schltr.

Sophronitis coccinea (Lindl.) Reichb.

Stelis cf. fraterna Lindl.

Indeterminada 1

Indeterminada 2

Indeterminada 3

Indeterminada 4

Indeterminada 5

OROBANCHACEAE

Velloziella westermanii Dusén

\section{PASSIFLORACEAE}

Passiflora mendoncaei Harms

\section{PENTAPHYLLACACEAE}

Ternstroemia brasiliensis Cambess

\section{PIPERACEAE}

Peperomia $\mathrm{cf}$. rizzinii Yunker

Peperomia cf. trineuroides Dahlst.

Peperomia corcovadensis Gardner

Peperomia sp. 1

Peperomia sp. 2

Peperomia tetraphylla (G. Forst.) Hook. \& Arn.

Piper xylosteoides (Kunth) Steud.

\begin{tabular}{|c|c|c|c|c|c|c|}
\hline ARV & & & $X$ & $X$ & $\mathrm{~T}$ & AYM 186 \\
\hline TREP & $X$ & $X$ & $\mathrm{X}$ & & $\mathrm{T}$ & AYM 144 \\
\hline EPI & & & & $X$ & $\mathrm{TM}$ & MBS 580 \\
\hline EPI & & & & $\mathrm{X}$ & TM & AYM 208 \\
\hline EPI & & & & $X$ & TM & AYM 209 \\
\hline EPI & & $X$ & & & $\mathrm{~T}$ & AYM 240 \\
\hline HER & & & & $X$ & $\mathrm{TC}$ & MBS 113 \\
\hline EPI & & & & $X$ & $\mathrm{TM}$ & AYM 211 \\
\hline EPI & & & & $X$ & $\mathrm{TM}$ & AYM 212 \\
\hline EPI & & & & $X$ & $\mathrm{TM}$ & AYM 131 \\
\hline EPI & & & & & $\mathrm{T}$ & MBS 608 \\
\hline EPI & & & & $X$ & $\mathrm{~T}$ & MBS s $/ \mathrm{n}^{\circ}$ \\
\hline EPI & & & & & $\mathrm{T}$ & MBS 577 \\
\hline HER & & & & $X$ & TM & MBS 581 \\
\hline HER & & & & $X$ & $\mathrm{~T}$ & MBS $s / n^{\circ}$ \\
\hline EPI & & $\mathrm{X}$ & & & $\mathrm{T}$ & AYM 206 \\
\hline EPI & & & & $X$ & $\mathrm{TM}$ & MBS 582 \\
\hline EPI & & & & $X$ & $\mathrm{TM}$ & AYM 239 \\
\hline EPI & & & & $X$ & $\mathrm{TM}$ & MBS $s / n^{\circ}$ \\
\hline HER & $X$ & & $\mathrm{X}$ & $X$ & $\mathrm{TM}$ & AYM 678 \\
\hline EPI & & & & & $\mathrm{T}$ & AYM 189 \\
\hline EPI & & $X$ & & & $\mathrm{~T}$ & MBS $s / n^{\circ}$ \\
\hline HER & & & & $\mathrm{X}$ & TM & MBS 578 \\
\hline EPI & & & & $X$ & $\mathrm{TM}$ & AYM 188 \\
\hline EPI & $X$ & $\mathrm{X}$ & $\mathrm{X}$ & $X$ & $\mathrm{~T}$ & MBS 114 \\
\hline EPI & & & & & $\mathrm{T}$ & AYM 238 \\
\hline EPI & & $X$ & & & $\mathrm{~T}$ & MBS $s / n^{\circ}$ \\
\hline EPI & & & $\mathrm{X}$ & & $\mathrm{T}$ & MBS $\mathrm{s} / \mathrm{n}^{\circ}$ \\
\hline EPI & & & & $X$ & $\mathrm{TM}$ & MBS $s / n^{\circ}$ \\
\hline EPI & & & & $X$ & $\mathrm{TM}$ & $\operatorname{MBS~s} / \mathrm{n}^{\circ}$ \\
\hline HER & & & & $X$ & $\mathrm{TM}$ & MBS 578 \\
\hline $\mathrm{ARB}$ & $X$ & & $X$ & $X$ & $\mathrm{~T}$ & MBS 598 \\
\hline TREP & $X$ & $X$ & & & $\mathrm{~T}$ & AYM 193 \\
\hline ARV & $X$ & $\mathrm{X}$ & $X$ & $X$ & $\mathrm{~T}$ & $\operatorname{MBS~s} / \mathrm{n}^{\circ}$ \\
\hline HER & $X$ & $X$ & & & $\mathrm{~T}$ & MBS 564 \\
\hline HER & & & & & $\mathrm{T}$ & MBS 565 \\
\hline HER & $X$ & $\mathrm{X}$ & & & $\mathrm{T}$ & MBS 414 \\
\hline HER & $X$ & & & & $\mathrm{~T}$ & MBS 689 \\
\hline HER & $X$ & $X$ & & & $\mathrm{~T}$ & AYM 312 \\
\hline HER & $X$ & & & $X$ & $\mathrm{~T}$ & AYM 195 \\
\hline ARB & & $\mathrm{X}$ & & & $\mathrm{T}$ & MBS 563 \\
\hline
\end{tabular}

Fitofisionomia Amostra

Local IGR PRA GIG

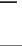

(1)

\author{
Amostra
}


Tabela 3. Continuação...

\section{Família/Espécie}

POACEAE

Aulonemia fimbriatifolia M.G.Clark

Chusquea anelytroides Rupr. ex. Doell

Paradiolyra micrantha (Kunth) Davidse \& Zuloaga

Panicum sp.

Panicum sp.

Paspalum polyphyllum Nees ex Trin.

Indeterminada 1

Indeterminada 2

Indeterminada 3

Indeterminada 4

Indeterminada 5

Indeterminada 6

POLIGONACEAE

Coccoloba persicaria L.

\section{PROTEACEAE}

Euplassa cantareirae Sleumer

Euplassa aff. nebularis Rambo \& Sleumer

Roupala rhombifolia Mart. ex Meissn.

Roupala cf. consimilis $\mathrm{Mez}$

RHAMNACEAE

Rhamnus sphaerosperma $\mathrm{Sw}$.

\section{ROSACEAE}

Prunus brasiliensis (Cham. \& Schltdl.) Dietrich

\section{RUBIACEAE}

Alibertia concolor (Cham.) Schum.

Coccocypselum condalia Pers.

Coccocypselum guianense (Aubl.) K. Schum.

Coussarea contracta Benth. \& Hook.f.

Faramea cf. cyanea Müll. Arg.

Faramea sp. 1

Faramea sp. 2

Galium hypocarpium (L.) Endl. ex Griseb ssp. indecorum

(Cham. \& Scl.) Dempster

Manettia cordifolia Wart.

Psychotria stachyoides Benth.

Psychotria sp. 1

Psychotria sp. 2

Rudgea jasminoides (Cham.) Müll. Arg

Rudgea parquioides (Cham.) Müll. Arg.

Rudgea sp.

Indeterminada 1

Indeterminada 2

SALICACEAE

Xylosma pseudosalzmanii Sleumer

\section{SAPINDACEAE}

Matayba guianensis Aubl.

SMILACACEAE

Smilax campestris Griseb.
Forma

\begin{tabular}{lll}
\multicolumn{3}{c}{ Local } \\
\hline IBI & IGR PRA & GIG
\end{tabular}

Fitofisionomia

Amostra

$\begin{array}{ll}\text { HER } & X \\ \text { HER } & X \\ \text { HER } & X \\ \text { HER } & X \\ \text { HER } & X \\ \text { HER } & \\ \text { HER } & \\ \text { TREP } & \\ \text { HER } & \\ \text { HER } & \\ \text { HER } & \\ \text { HER } & \end{array}$

ARB

$$
\text { X }
$$

X

$\mathrm{T}$

ARV X

ARV $X$

ARV

ARV

X

$\mathrm{X}$

ARV

X X

ARV

X X

ARV

HER

HER

ARV

ARV

ARV

ARV

HER

TRE

ARB

ARB

ARB

ARB

ARV

ARB

ARB

ARB

$X$
$X$

$X$
$X$

TC

MBS 456

AYM 301

MBS 683

AYM 300

MBS $\mathrm{s} / \mathrm{n}^{\circ}$

MBS 472

MBS 469

MBS 677

AYM 108

AYM 18

MBS 471

AYM 300

AYM 200

MBS $s / n^{\circ}$

MBS 465

MBS 502

MBS $\mathrm{s} / \mathrm{n}^{\circ}$

AYM 203

MBS 574

AYM 80

MBS 329

MBS 294

AYM 132

AYM 83

AYM 80

AYM 242

MBS 303

AYM 29

MBS 597

AYM 82

MBS 266

AYM 228

MBS 68

MBS 301

MBS 68

MBS 446

ARV $\quad X \quad X$

$\mathrm{T}$

AYM 180

ARV $\quad \mathrm{X} \quad$ TM

AYM 229 
Tabela 3. Continuação...

\begin{tabular}{|c|c|c|c|c|c|c|c|}
\hline \multirow[t]{2}{*}{ Família/Espécie } & \multirow[t]{2}{*}{ Forma } & \multicolumn{4}{|c|}{ Local } & \multirow[t]{2}{*}{ Fitofisionomia } & \multirow[t]{2}{*}{ Amostra } \\
\hline & & IBI & IGR & PRA & GIG & & \\
\hline \multicolumn{8}{|l|}{ SOLANACEAE } \\
\hline Brunfelsia cf. pilosa Plowman & $\mathrm{ARB}$ & $\mathrm{X}$ & & & & $\mathrm{T}$ & MBS 599 \\
\hline Solanum megalochiton Mart. & TREP & & & & $\mathrm{X}$ & $\mathrm{TM}$ & AYM 23 \\
\hline Solanum sp. 1 & $\mathrm{ARB}$ & $\mathrm{X}$ & & & & $\mathrm{T}$ & MBS 695 \\
\hline \multicolumn{8}{|l|}{ STYRACACEAE } \\
\hline Styrax martii Seub. & ARV & & $\mathrm{X}$ & & $\mathrm{X}$ & $\mathrm{TM}$ & AYM 85 \\
\hline \multicolumn{8}{|l|}{ SYMPLOCACEAE } \\
\hline Symplocos incrassata Aranha & ARV & $\mathrm{X}$ & & & & $\mathrm{T}$ & AYM 87 \\
\hline Symplocos corymboclados Brand & ARV & $\mathrm{X}$ & $\mathrm{X}$ & $\mathrm{X}$ & $\mathrm{X}$ & $\mathrm{T}$ & MBS 701 \\
\hline Symplocos bidana Aranha & ARV & & $\mathrm{X}$ & $\mathrm{X}$ & $\mathrm{X}$ & $\mathrm{T}$ & AYM 24 \\
\hline \multicolumn{8}{|l|}{ THEACEAE } \\
\hline Gordonia fruticosa (Schrad.) H.Keng & ARV & $\mathrm{X}$ & $\mathrm{X}$ & $\mathrm{X}$ & $\mathrm{X}$ & $\mathrm{T}$ & AYM 88 \\
\hline \multicolumn{8}{|l|}{ THYMELAEACEAE } \\
\hline Daphnopsis fasciculata (Meisn.) Nevling & ARV & & & & $\mathrm{X}$ & $\mathrm{TC}$ & AYM 235 \\
\hline Daphnopsis sellowiana Taub. & ARV & $\mathrm{X}$ & $\mathrm{X}$ & $\mathrm{X}$ & & $\mathrm{T}$ & MBS 605 \\
\hline \multicolumn{8}{|l|}{ VALERIANACEAE } \\
\hline Valeriana ulei Grabn. & HER & $\mathrm{X}$ & $\mathrm{X}$ & $\mathrm{X}$ & & $\mathrm{TC}$ & MBS 73 \\
\hline \multicolumn{8}{|l|}{ VIOLACEAE } \\
\hline Viola cerasifolia A.St.-Hill. & HER & $\mathrm{X}$ & & $\mathrm{X}$ & & $\mathrm{T}$ & AYM 237 \\
\hline \multicolumn{8}{|l|}{ VOCHYSIACEAE } \\
\hline Vochysia cf. bifalcata Warm. & ARV & & & & $\mathrm{X}$ & $\mathrm{TM}$ & AYM 246 \\
\hline \multicolumn{8}{|l|}{ WINTERACEAE } \\
\hline Drimys angustifolia Miers. & ARV & $X$ & $\mathrm{X}$ & $\mathrm{X}$ & & $\mathrm{T}$ & AYM 236 \\
\hline Drimys brasiliensis Miers. & ARV & $\mathrm{X}$ & $\mathrm{X}$ & $X$ & $\mathrm{X}$ & $\mathrm{T}$ & MBS 606 \\
\hline \multicolumn{8}{|l|}{ PODOCARPACEAE } \\
\hline Podocarpus sellowii Klotzsch & ARV & $X$ & $\mathrm{X}$ & $\mathrm{X}$ & $\mathrm{X}$ & $\mathrm{T}$ & MBS 231 \\
\hline \multicolumn{8}{|l|}{ ASPLENIACEAE } \\
\hline Asplenium oligophyllum Kaulf. & EPI & $X$ & & & & $\mathrm{~T}$ & AYM 345 \\
\hline Asplenium scandicinum Kaulf. & EPI & & $\mathrm{X}$ & & & $\mathrm{T}$ & MBS 725 \\
\hline Asplenium harpeodes Kunze & EPI & $X$ & & & & $\mathrm{~T}$ & MBS 726 \\
\hline Asplenium pseudonitidum Raddi & EPI & $\mathrm{X}$ & & & & $\mathrm{T}$ & AYM 91 \\
\hline Indeterminada 1 & HER & & & $\mathrm{X}$ & & $\mathrm{T}$ & AYM 147 \\
\hline \multicolumn{8}{|l|}{ BLECHNACEAE } \\
\hline Blechnum cordatum (Desv.) Hieron. & HER & $\mathrm{X}$ & $\mathrm{X}$ & & $\mathrm{X}$ & $\mathrm{T}$ & AYM 10 \\
\hline \multicolumn{8}{|l|}{ DRYOPTERIDACEAE } \\
\hline Arachniodes cf. denticulata (Sw.) Ching & EPI & $\mathrm{X}$ & $\mathrm{X}$ & & & $\mathrm{T}$ & MBS 743 \\
\hline Pteris deflexa Link & HER & $\mathrm{X}$ & & & & $\mathrm{T}$ & MBS 732 \\
\hline Rumohra adiantiformis (G. Forst.) Ching & HER & $\mathrm{X}$ & $\mathrm{X}$ & $\mathrm{X}$ & & $\mathrm{T}$ & MBS 706 \\
\hline Lastreopsis amplissima (C. Presl) Tindale & & & $X$ & $\mathrm{X}$ & & $\mathrm{T}$ & AYM 306 \\
\hline Indeterminada 1 & HER & & $\mathrm{X}$ & & & $\mathrm{T}$ & AYM 330 \\
\hline \multicolumn{8}{|l|}{ CYATHECEAE } \\
\hline Alsophila capensis J. Sm. & HER & & $\mathrm{X}$ & & & $\mathrm{T}$ & MBS 723 \\
\hline Cyathea corcovadensis (Raddi) Domin & HER & & & & $\mathrm{X}$ & $\mathrm{TM}$ & AYM 343 \\
\hline Cyathea sp. 1 & HER & & & $\mathrm{X}$ & $\mathrm{X}$ & $\mathrm{T}$ & AYM 9 \\
\hline Cyathea sp. 2 & HER & $\mathrm{X}$ & & & & $\mathrm{T}$ & AYM 92 \\
\hline Cyathea sp. 3 & HER & $\mathrm{X}$ & & & & $\mathrm{T}$ & AYM 344 \\
\hline Plagiogyria fialhoi Copel & HER & $\mathrm{X}$ & & & & $\mathrm{T}$ & AYM 310 \\
\hline \multicolumn{8}{|l|}{ GLEICHENIACEAE } \\
\hline Gleichenella pectinata (Willd.) Ching & HER & & & & $\mathrm{X}$ & $\mathrm{TM}$ & AYM 356 \\
\hline
\end{tabular}




\begin{tabular}{|c|c|c|c|c|c|c|c|}
\hline \multirow[t]{2}{*}{ Família/Espécie } & \multirow[t]{2}{*}{ Forma } & \multicolumn{4}{|c|}{ Local } & \multirow[t]{2}{*}{ Fitofisionomia } & \multirow[t]{2}{*}{ Amostra } \\
\hline & & IBI & IGR & PRA & GIG & & \\
\hline \multicolumn{8}{|l|}{ GRAMMITIDACEAE } \\
\hline Cochlidium punctatum (Raddi) L.E. Bishop & & $\mathrm{X}$ & $\mathrm{X}$ & $\mathrm{X}$ & & $\mathrm{T}$ & AYM 332 \\
\hline $\begin{array}{l}\text { Lellingeria apiculata (Kunze ex Klotzsch) A.R. Sm. \& } \\
\text { R.C. Moran }\end{array}$ & EPI & & $\mathrm{X}$ & & & $\mathrm{T}$ & MBS 711 \\
\hline Lellingeria depressa (C. Chr.) A.R. Sm. \& R.C. Moran & EPI & & $\mathrm{X}$ & & $\mathrm{X}$ & $\mathrm{T}$ & AYM 338 \\
\hline Lellingeria organensis (Gardner) A.R. Sm. \& R.C. Moran & EPI & $\mathrm{X}$ & & & & $\mathrm{T}$ & MBS 715 \\
\hline Terpsichore achilleifolia (Kaulf.) A.R. Sm. & EPI & $\mathrm{X}$ & $\mathrm{X}$ & $\mathrm{X}$ & & $\mathrm{T}$ & AYM 14 \\
\hline Terpsichore reclinata (Branc) Labiak & EPI & $\mathrm{X}$ & & & & $\mathrm{T}$ & MBS 733 \\
\hline \multicolumn{8}{|l|}{ HYMENOPHYLLACEAE } \\
\hline Hymenophyllum asplenioides (Sw.) Sw. & HER & & $\mathrm{X}$ & & $\mathrm{X}$ & $\mathrm{T}$ & MBS 720 \\
\hline Hymenophyllum caudiculatum Mart. & HER & $\mathrm{X}$ & & & & $\mathrm{T}$ & MBS 719 \\
\hline Hymenophyllum magellanicum Willd. & HER & $\mathrm{X}$ & $\mathrm{X}$ & & & $\mathrm{T}$ & AYM 342 \\
\hline Hymenophyllum polyanthos (Sw.) Sw. & HER & $\mathrm{X}$ & $\mathrm{X}$ & & & $\mathrm{T}$ & MBS 718 \\
\hline Hymenophyllum pulchellum Schltdl. \& Cham. & HER & & & & $\mathrm{X}$ & $\mathrm{T}$ & AYM 339 \\
\hline Trichomanes cristatum Kaulf. & HER & & & & $\mathrm{X}$ & $\mathrm{T}$ & MBS 721 \\
\hline \multicolumn{8}{|l|}{ LOMARIOPSIDACEAE } \\
\hline Elaphoglossum ornatum (Mett. ex Kuhn) H. Christ & EPI & $\mathrm{X}$ & $\mathrm{X}$ & & & $\mathrm{T}$ & AYM 336 \\
\hline Elaphoglossum squamipes (Hook.) T.Moore & EPI & & $\mathrm{X}$ & $\mathrm{X}$ & & $\mathrm{T}$ & AYM 16 \\
\hline \multicolumn{8}{|l|}{ LYCOPODIACEAE } \\
\hline Huperzia acerosa (Sw.) Holub & EPI & & & & $\mathrm{X}$ & TM & AYM 349 \\
\hline Huperzia heterocarpon (Fée) Holub & EPI & $\mathrm{X}$ & $\mathrm{X}$ & $\mathrm{X}$ & & $\mathrm{T}$ & MBS 390 \\
\hline Huperzia quadrifariata (Bory ex Duperrey) Rothm. & EPI & $\mathrm{X}$ & $\mathrm{X}$ & $\mathrm{X}$ & & $\mathrm{T}$ & MBS 703 \\
\hline \multicolumn{8}{|l|}{ POLYPODIACEAE } \\
\hline Campyloneurum cf. acrocarpon & EPI & & & & $\mathrm{X}$ & $\mathrm{TM}$ & AYM 335 \\
\hline Campyloneurum fallax Fée & EPI & $\mathrm{X}$ & & & & $\mathrm{T}$ & MBS 458 \\
\hline Campyloneurum nitidum (Kaulf.) C. Presl & EPI & $\mathrm{X}$ & $\mathrm{X}$ & $\mathrm{X}$ & & $\mathrm{T}$ & MBS 713 \\
\hline Pecluma sicca (Lindm.) M.G. Price & EPI & & $\mathrm{X}$ & & & $\mathrm{T}$ & MBS 711 \\
\hline Pecluma recurvata (Kaulf.) M.G. Price & EPI & & $\mathrm{X}$ & & & $\mathrm{T}$ & MBS 740 \\
\hline Pecluma sp. & EPI & & & $\mathrm{X}$ & & $\mathrm{T}$ & AYM 305 \\
\hline Polypodium hirsutissimum Raddi & EPI & $\mathrm{X}$ & & $\mathrm{X}$ & $\mathrm{X}$ & $\mathrm{T}$ & AYM 359 \\
\hline Serpocaulon catharinae (Langsd. \& Fisch.) A.R. Sm. & EPI & & & $\mathrm{X}$ & $\mathrm{X}$ & $\mathrm{T}$ & MBS 741 \\
\hline \multicolumn{8}{|l|}{ PTERIDACEAE } \\
\hline Adianthum sp. 1 & EPI & $\mathrm{X}$ & & & & $\mathrm{T}$ & AYM 334 \\
\hline Adianthum sp. 2 & HER & & & & $\mathrm{X}$ & $\mathrm{TM}$ & AYM 333 \\
\hline Histiopteris incisa (Thunb.) J. Sm. & HER & & & & $\mathrm{X}$ & $\mathrm{T}$ & AYM 17 \\
\hline Lindsaea botrychioides A. St.-Hil. & HER & $\mathrm{X}$ & & & & $\mathrm{T}$ & MBS 702 \\
\hline Lindsaea ovoidea Fée & HER & & & & $\mathrm{X}$ & $\mathrm{T}$ & MBS 709 \\
\hline Pteris sp. & HER & & & & & $\mathrm{T}$ & MBS 724 \\
\hline \multicolumn{8}{|l|}{ SCHIZAEACEAE } \\
\hline Anemia ferruginea Kunth & HER & $\mathrm{X}$ & & & & $\mathrm{T}$ & MBS 712 \\
\hline \multicolumn{8}{|l|}{ SELAGINELLACEAE } \\
\hline Selaginella macrostachya (Spring) Spring & HER & & & & $\mathrm{X}$ & $\mathrm{TM}$ & MBS 735 \\
\hline Selaginella sp. 1 & HER & & & & $\mathrm{X}$ & $\mathrm{TM}$ & AYM 354 \\
\hline \multicolumn{8}{|l|}{ THELYPTERIDACEAE } \\
\hline Thelypteris sp. & HER & $\mathrm{X}$ & $X$ & $X$ & $\mathrm{X}$ & $\mathrm{T}$ & AYM 149 \\
\hline Indeterminada 1 & HER & $\mathrm{X}$ & & & & $\mathrm{T}$ & AYM 310 \\
\hline Indeterminada 2 & HER & & & $\mathrm{X}$ & & $\mathrm{T}$ & AYM 147 \\
\hline Indeterminada 3 & HER & & & & $\mathrm{X}$ & TM & AYM 343 \\
\hline Indeterminada 4 & HER & $X$ & & & & $\mathrm{~T}$ & AYM 344 \\
\hline Total & & 185 & 165 & 129 & 154 & & \\
\hline
\end{tabular}


Nas florestas altomontanas deste estudo foram encontradas espécies novas, descritas recentemente e encontradas também em refúgios vegetacionais altomontanos por Mocochinski \& Scheer (2008), tais como Alstroemeria amabilis (Assis 2003), Aulonemia fimbriatifolia (Clark 2004) e Myrsine altomontana (Freitas \& Kinoshita 2005). Além destas, 2 espécies de Symplocos descritas recentemente (Aranha et al. 2009) e uma espécie nova de Hesperozigis (Figura 2), ainda não descrita (Tabela 3) também foram encontradas nas florestas altomontanas pelo presente trabalho. Esta situação reflete o pequeno esforço de pesquisa realizado nos ecossistemas altomontanos e evidencia a importância de ações para sua conservação.

Considerando a riqueza florística vascular das florestas altomontanas na Serra do Mar no Paraná apresentada neste trabalho, juntamente com o levantamento da flora vascular dos campos altomontanos realizado por Mocochinski \& Scheer (2008), estabelece-se uma aproximação da riqueza florística dos ecossistemas altomontanos na Serra do Mar paranaense. Compilando-se as listas florísticas das duas formações (florestas altomontanas e refúgios vegetacionais altomontanos), obtem-se um total de 507 espécies ocorrentes nos ecossistemas altomontanos da Serra do Mar no Paraná.

\section{Comparações da florística vascular das florestas amostradas}

As florestas altomontanas da Serra do Ibitiraquire apresentaram maior riqueza de espécies (185), seguidas das Serras da Igreja (165), Serra Gigante (154) e Serra da Prata (129). O número de espécies arbóreas detectadas foram 66, 56, 60 e 49, respectivamente. Das espécies listadas, 35 foram comuns às quatro serras, sendo 23 arbóreas (Tabela 3 ).

O dendrograma da análise de agrupamento resultante da presença e ausência de espécies vasculares nas florestas altomontanas das quatro serras estudadas (Figura 4) indicou uma maior proximidade florística entre as serras da Igreja e da Prata. A Serra do Ibitiraquire ficou em posição intermediária e a Serra Gigante apresentou menor proximidade. Os índices de similaridade de Sörensen reforçam tais

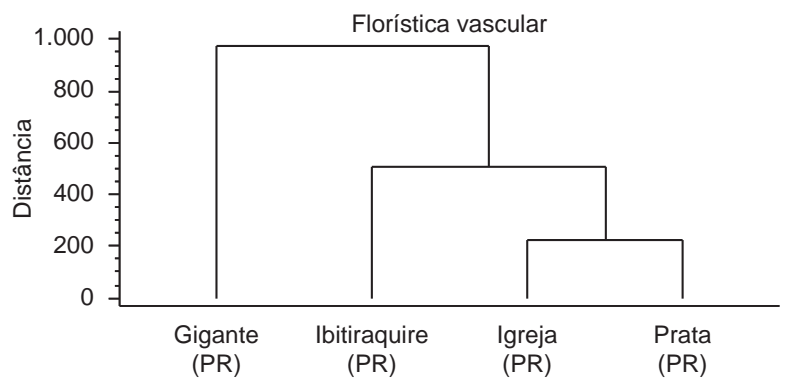

Figura 4. Dendrograma de análise de agrupamento com base na presença e ausência de espécies vasculares nas florestas altomontanas de quatro serras amostradas no presente estudo.

Figure 4. Cluster analysis dendrogram of vascular species presence and absence in the four cloud forests sampled in the present study, Southern Brazil. resultados (Tabela 4). Além de serem mais próximas entre si (aproximadamente $20 \mathrm{~km}$ ), as florestas altomontanas das serras da Igreja e da Prata apresentam altitudes e gradientes altitudinais similares, entre 1.250 e 1.480 m s.n.m.. A Serra do Ibitiraquire apresenta maior extensão e maior gradiente altitudinal, com florestas altomontanas típicas, em alguns locais ocorrendo desde os $1.200 \mathrm{~m}$, alcançando os 1.850 m s.n.m., justificando sua maior riqueza de espécies (Tabela 3). A Serra Gigante apresenta florestas altomontanas em menores altitudes (entre 950 e 1.050 m s.n.m.), patamares considerados montanos nas outras serras amostradas. A ocorrência de florestas tipicamente altomontanas em menores altitudes, em montanhas pequenas e isoladas, geralmente mais próximas ao mar e constantemente cobertas por neblina, é comumente atribuída ao efeito de elevação de massa ou "Massenerhebung effect", fenômeno que envolve condições geográficas, geomorfológicas e climáticas, as quais tem influência na temperatura, na formação de nuvens e na insolação ultravioleta (Grubb 1971, Flenley 1995). Tais florestas estudadas no presente trabalho (Serra Gigante), apresentam uma área mais restrita e com um menor gradiente altitudinal, sofrem uma maior influência de ecossistemas montanos, justificando sua menor similaridade com os outros trechos amostrados.

\section{Comparações com outras serras do sul e sudeste brasileiro}

Segundo Tabela 3, nenhuma espécie arbórea foi comum a todas as áreas comparadas. Os índices de similaridade de Sörensen variaram de 0,04 até 0,78 (Tabela 5).

O dendrograma da análise de agrupamento resultante da presença e ausência de espécies arbóreas nas florestas altomontanas das quatro serras estudadas e outras florestas ocorrentes acima dos $1.380 \mathrm{~m}$ de altitude na região sul (PR e SC) e acima dos 1.800 m na região sudeste (SP, RJ e MG) do Brasil, destacou dois grupos distintos (Figura 5).

O primeiro grupo incluiu as serras amostradas no presente trabalho e também as outras serras paranaenses compiladas na Tabela 2, todas classificadas como "Floresta Ombrófila Densa Altomontana" e pertencentes ao Complexo Serra do Mar. Das serras amostradas, a Serra Gigante indicou ligeiramente maior proximidade com os trabalhos realizados em altitudes mais baixas na Serra da Baitaca (pico Anhangava) e na Serra do Marumbi (picos Marumbi e Vigia), em torno dos 1.280 m s.n.m.. Nesse grupo, seis espécies arbóreas foram encontradas em todas as áreas: Ilex microdonta, Drimys brasiliensis, Gomidesia sellowiana, Blepharocalix salicifolius, Gordonia fruticosa e Ocotea porosa (considerada como $O$. catharinensis na compilação de Koehler et al. 2002).

O segundo grupo incluiu tanto florestas altomontanas representativas dos estados mais ao sul (SC e RS) quanto mais ao norte (SP, RJ e MG) das florestas do presente trabalho. No entanto, as duas florestas altomontanas de SC mostraram maior dissimilaridade com as outras dentro desse grupo. Os índices de similaridade de Sörensen reforçam tais resultados (Tabela 5). Isto está provavelmente ligado à grande distância entre as áreas, resultando em influências de diferentes centros de endemismo, como por exemplo o centro São Paulo/Rio de

Tabela 4. Similaridades florísticas com base no índice de Sörensen, considerando as espécies vasculares (incluindo determinações em nível de gênero e de morfotipos) das florestas altomontanas das quatro serras amostradas no Paraná.

Table 4. Vascular Floristic Sörensen similarities for four cloud forests in the state of Paraná, Southern Brazil.

\begin{tabular}{|c|c|c|c|c|}
\hline & Ibitiraquire (PR) & & & \\
\hline Ibitiraquire (PR) & - & Igreja (PR) & & \\
\hline Igreja (PR) & 0,57 & - & Prata (PR) & \\
\hline Prata (PR) & 0,60 & 0,62 & - & Gigante (PR) \\
\hline Gigante (PR) & 0,34 & 0,39 & 0,40 & - \\
\hline
\end{tabular}




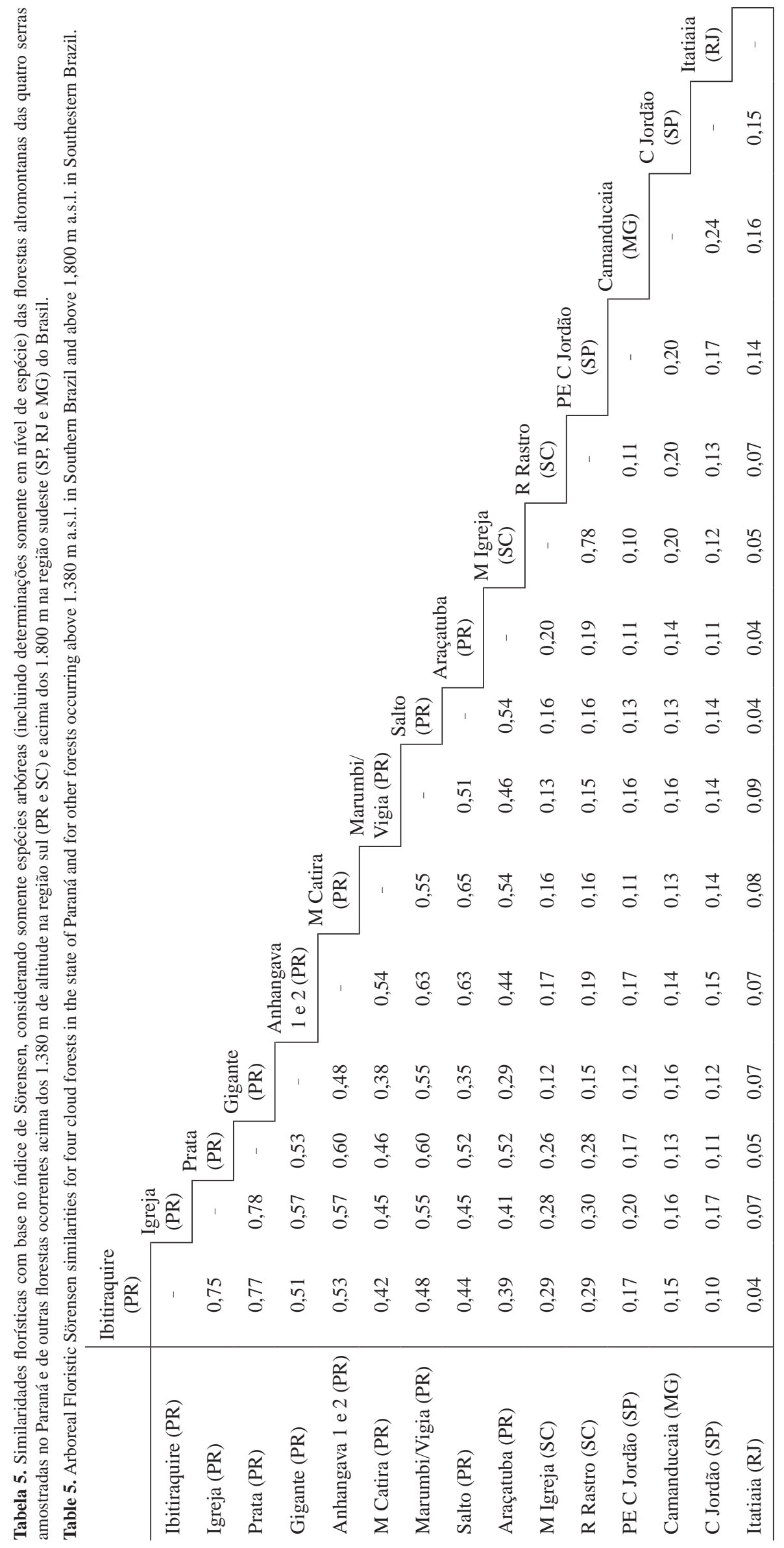




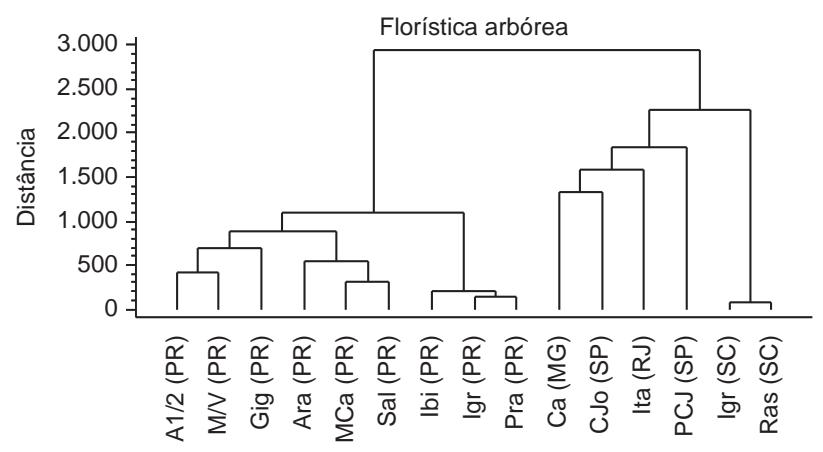

Figura 5. Dendrograma de análise de agrupamento com base na presença e ausência de espécies arbóreas nas quatro serras amostradas no Paraná e de outras florestas ocorrentes acima dos $1.380 \mathrm{~m}$ de altitude na região sul (PR e $\mathrm{SC}$ ) e acima dos $1.800 \mathrm{~m}$ na região sudeste (SP, RJ e MG) do Brasil.

Figure 5. Cluster analysis dendrogram of vascular species presence and absence in the four cloud forests sampled in the present study and for other forests occurring above 1,380 m a.s.l. in Southern Brazil and above $1.800 \mathrm{~m}$ a.s.l. in Southestern Brazil.

Janeiro, discutido por Thomas et al. (1998), que tem maior influência nas regiões costeiras dos estados de São Paulo, Rio de Janeiro e sul do Espírito Santo.

$\mathrm{Na}$ análise realizada, as florestas altomontanas paranaenses ( $1^{\circ}$ grupo) somente apresentaram Ilex microdonta como espécie arbórea comum com as áreas de SC (índices de similaridade entre $0,12 \mathrm{e}$ 0,29; Tabela 5). O $1^{\circ}$ grupo apresentou somente Drimys brasiliensis como espécie arbórea comum em todas as áreas comparadas mais ao norte (SP, RJ e MG) (índices de similaridade entre 0,04 e 0,20; Tabela 5).

Uma possível explicação para a diferenciação dos dois grupos é que a maior parte dos trabalhos em São Paulo, Rio de Janeiro e Santa Catarina indicou uma forte ocorrência de elementos da Floresta Ombrófila Mista, tais como Araucaria angustifolia e Podocarpus lambertii, o que não ocorre nas áreas altomontanas estudadas do Paraná (Floresta Ombrófila Densa Altomontana). Neste estado, tais espécies somente ocorrem no ecótono Floresta Ombrófila Densa/ Mista, situado no patamar montano da face oeste da Serra do Mar, em contato com o planalto. Historicamente, a Araucaria angustifolia tem seu ponto mais setentrional (formação disjunta) na serra do Caparaó (Complexo da Serra da Mantiqueira), próximo à fronteira de Minas Gerais e do Espírito Santo, local alcançado em períodos climáticos favoráveis do Quaternário (Leite 2002). A presença desses elementos na região provavelmente deve-se ao relevo, aos solos, às menores temperaturas resultantes da altitude, e a menor influência das massas de ar e umidade oceânicas, mais comuns na face oriental da Serra do Mar (Oliveira-Filho \& Fontes 2000). Além disso, as florestas consideradas nos outros trabalhos foram descritas como secundárias e/ou alteradas por pastagens e/ou queimadas, incluindo citações de espécies comuns nas fases iniciais e intermediárias de sucessão secundária e em patamares montanos (por exemplo: Achornea glandulosa, Croton urucurana, Piptocarpha angustifolia, Mimosa scabrella e Myrsine coriacea).

Outros fatores que diferem tais florestas são as formações geológicas e geomorfológicas diferentes das serras descritas no Paraná (Tabela 1). Por exemplo, a formação basáltica das serras do Rio do Rastro e da Igreja em Santa Catarina, (Falkenberg 2003), apresenta feições geomorfológicas diferentes das originárias das formações predominantemente graníticas da Serra do Mar paranaense. Tais condições resultaram em solos com diferentes profundidades e com características físico-químicas diferentes, que obviamente interferem na estrutura e composição da floresta. As florestas sobre Latossolos em Camanducaia, em Minas Gerais (França \& Stehmann 2004), apresentam em média, $17 \mathrm{~m}$ de altura, diferindo das demais florestas analisadas neste trabalho (alturas entre 3 e $7 \mathrm{~m}$ ) e concordando com a descrição de florestas altomontanas de Veloso et al. (1991).

Portanto, ainda existem poucas informações para melhor definir e comparar as florestas altomontanas brasileiras, nas suas diferentes formações fitogeográficas: Forestas Ombrófilas Densas (Amazônica e Atlântica), Ombrófila Mista e Estacionais. Para se obter melhores parâmetros sobre riqueza, distribuição de espécies e endemismo, são necessários trabalhos que priorizem a florística vascular de florestas altomontanas primárias e bem conservadas. Novos conhecimentos dessas formações e de suas funções, principalmente àquelas vinculadas à sua importância hidrológica, devem ser apresentados à sociedade e subsidiar melhor, medidas para sua conservação, manejo e restauração.

\section{Agradecimentos}

Agradecemos à Fundação O Boticário de Proteção à Natureza pelo patrocínio do "Projeto Altomontana - A Floresta Ombrófila Densa Altomontana e os Refúgios Vegetacionais Altomontanos no Paraná”, à Sociedade Fritz Muller de Ciências Naturais pelo apoio ao projeto. Agradecemos também às contribuições de Ruddy Proença, Samuel Arruda, Sandro M. Silva, Gustavo Gatti, Gilberto Tiepolo, Carlos V. Roderjan, Osmar S. Ribas, Gert G. Hatschbach, Juarez Cordeiro, Edimilson Costa, Marcos Sobral, Renato Goldenberg, Paulo Labiak, Armando C. Cervi, Élide dos Santos, Olavo A. Guimarães, Marília Borgo, Miriam Kaehler, Marcelo Brotto, Fabrício Meyer, Carina Kozera, Rodrigo A. Kersten e dos revisores anônimos da revista.

\section{Referências Bibliográficas}

ALMEIDA, F.F.M. \& De CARNEIRO, C.D. 1998. Origem e evolução da Serra do Mar. Rev. Bras. Geocienc. 28(2):135-150.

Angiosperm Phylogeny Group - APG II. 2003. An update of the Angiosperm Phylogeny Group classification for the orders and families of flowering plants: APG II. Bot. j. Linn. Soc. 141(4):399-436.

ARANHA-FILHO, J.L.M., ALMEDA, FRITSCH, P.W., ALMEDA, F. \& MARTINS, A.B. 2009. Two new dioecious species of Symplocos (Symplocaceae) from Southern Brazil. Novon 19(1):1-6.

ASSIS, M.C. 2003. Duas novas espécies de Alstroemeria L. (Alstroemeriaceae) para o Brasil. Acta Bot. Bras. 17(2):179-182.

BIGARELLA, J.J. 1978. A Serra do Mar e a porção oriental do estado do Paraná, um problema de segurança ambiental e nacional (contribuição à geografia, geologia e ecologia regional). SEP; ADEA, Curitiba.

BÒLOS, O., CERVI, A.C. \& HATSCHBACH, G. 1991. Estudios sobre la vegetación del Paraná (Brasil Meridional). Collect. Bot. 20:79-182.

BRADE, A.C. 1956. A flora do Parque Nacional de Itatiaia. Boletim do Parque Nacional do Itatiaia 5:7-85.

BROWER, J.E. \& ZAR, J.H. 1984. Field and laboratorymethods for general ecology. W.M.C. Brow, Dubuque.

BRUIJNZEEL, L.A. \& PROCTOR, J. 1995. Hydrology and biogeochemistry of tropical montane cloud forests: what do we really know? In Tropical montane cloud forests (L.S. Hamilton, J.O. Juvik \& F.N. Scatena, eds). Springer Verlag, New York, p. 38-78.

BRUIJNZEEL, L.A. Hydrology of tropical montane cloud forests: a reassessment. 2000. In Proceedings of the Second International Colloquium (J.S. Gladwell, ed.). UNESCO, Paris, p. 353-383.

CLARK, L.G. 2004. New species of Aulonemia and Chusquea (Poaceae: Bambusoideae: Bambuseae) from southeastern Brazil. Rev. Brasil. Bot. 27(1):31-36. 
DOUMENGE, C., GILMOUR, D., PEREZ, M.R. \& BLOCKHUS, J. 1995. Tropical montane cloud forests: conservation status and management issues. In Tropical montane cloud forests (L.S. Hamilton, J.O. Juvik \& F.N. Scatena, eds). Springer Verlag, New York, p. 24-37.

DUSÉN, P. 1955. Contribuições para a flora do Itatiaia. Boletim do Parque Nacional do Itatiaia. Serviço Florestal, Rio de Janeiro.

FALKENBERG, D.B. \& VOLTOLINI, J.C. 1995. The montane cloud forest in southern Brazil. In Tropical montane cloud forests (L.S. Hamilton, J.O. Juvik \& F.N. Scatena, eds). Springer Verlag, New York, p. 138-149.

FALKENBERG, D.B. 2003. Matinhas nebulares e vegetação rupícola dos Aparados da Serra Geral (SC/RS), sul do Brasil. Tese de Doutorado, Universidade de Campinas, Campinas.

FLENLEY, J.R. 1995. Cloud Forest, the Massenerhebung effect, and ultraviolet insolation. In Tropical montane cloud forests (L.S. Hamilton, J.O. Juvik \& F.N. Scatena, eds). Springer Verlag, New York, p. 150-155.

FONTES, M.A.L. 1997. Análise da composição florística das florestas nebulares do Parque estadual do Ibitipoca, Minas gerais, Brasil. Lavras. Dissertação de Mestrado, Universidade Federal de Lavras, Lavras.

FRANÇA G.S. \& STEHMANN, J.R. 2004. Composição florística e estrutura do componente arbóreo de uma floresta altimontana no município de Camanducaia, Minas Gerais, Brasil. Rev. Brasil. Bot. 27(1):19-30.

FREITAS, M.F. \& KINOSHITA, L.S. 2005. Novas espécies de Myrsine L. (Myrsinaceae) para o Brasil. Rodriguesia, 56(87):67-72.

GRUBB, P.J. 1971. Interpretation of the "Massenerhebung" effect on tropical mountains. Nature, 229(5279):44-45.

HAMILTON, L.S., JUVIK, J.O. \& SCATENA, F.N. 1995. The Puerto Rico tropical cloud forest symposium: introduction and workshop synthesis. In Tropical montane cloud forests (L.S. Hamilton, J.O. Juvik \& F.N. Scatena, eds). Springer Verlag, New York, p. 1-23.

Instituto Brasileiro de Geografia e Estatística - IBGE. 1992. Manual técnico da vegetação brasileira. Fundação Instituto Brasileiro de Geografia e Estatística; Depto. de Recursos Naturais e Estudos Ambientais, Rio de Janeiro. Manuais Técnicos de Geociências, $n^{\circ} 1$.

Instituto Brasileiro de Geografia e Estatística - IBGE. 2004. Mapa da vegetação do Brasil. Escala 1:5.000.000. Disponível em: <ftp://geoftp.ibge.gov. $\mathrm{br} / \mathrm{mapas} /$ tematicos/mapas_murais/vegetacao.pdf $>$. (último acesso em 19/03/2009).

KLEIN, R.M. 1980. Ecologia da flora e vegetação do Vale do Itajaí. Sellowia, 31-32:9-389.

KOEHLER, A. 2001. Floresta ombrófila densa altomontana: aspectos florísticos e estruturais do componente arbóreo em diferentes trechos da serra do mar, PR. Dissertação de Mestrado, Universidade Federal do Paraná, Curitiba.

KOEHLER, A., GALVÃO, F. \& LONGHI, S.J. 2002. Floresta Ombrófila Densa Altomontana: aspectos florísticos e estruturais de diferentes trechos da serra do mar. Ci. Fl. 12(2):27-39.

LEITE, P.F. 2002. Contribuição ao conhecimento fitoecológico do sul do Brasil. Cienc. Ambient. 24(1):51-73.

MAACK, R. 2002. Geografia física do Estado do Paraná. Imprensa Oficial, Curitiba.

MEIRA NETO, J.A., BERNACCI, L.C., GROMBONE, M.T., TAMASHIRO, J.Y. \& LEITÃO-FILHO, H.F. 1989. Composição florística da floresta semidecídua de altitude no Parque Municipal da grota Funda (Atibaia, estado de São Paulo). Acta. Bot. Bras. 3:51-74.

MOCOCHINSKI, A.Y. \& SCHEER, M.B. 2008. Campos de altitude na serra do mar paranaense: aspectos florísticos. Rev. Floresta, 38(4):625-640.
OLIVEIRA-FILHO, A.T. \& FONTES, M.A.L. 2000. Patterns of floristic differentiation among Atlantic forests in southeastern Brazil and the influence of climate. Biotropica, 32(4b):793-810.

OLIVEIRA FILHO, A.T., CARVALHO, D.A., FONTES, M.A.L., BERG, E.V.D., CURI, N. \& CARVALHO, W.A.C. 2004. Variações estruturais do compartimento arbóreo de uma floresta semidecídua alto-montana na chapada dos Perdizes, Carrancas, MG. Rev. Brasil. Bot. 27(2):291-309.

PEREIRA-SILVA, E.F.L., HARDT, E., FRANCISCO, C.E.S. 2007. Caracterização florística da vegetação lenhosa de um fragmento urbano de Floresta Ombrófila Mista Alto Montana, Campos do Jordão, SP. Holos Environment, 7(2):154-170.

PETEAN, M.P. 2002. Epífitas vasculares em floresta altomontana, parque estadual pico do Marumbi, Morretes, PR. Dissertação de Mestrado, Universidade Federal do Paraná, Curitiba.

PORTES, M.C.G.O., GALVÃO, F. \& KOEHLER, A. 2001. Caracterização floristica e estrutural de uma Floresta Ombrófila Densa Altomontana do morro Anhangava, Quatro Barras, PR. Revista Floresta 31(1 e 2):22-31.

Programa de Proteção da Floresta Atlântica - PRÓ-ATLÂNTICA. 2002. Projeto Carta Geológica. Cartas: MI-2844-2 Ariri, MI-2843-1 Represa do Capivari, MI-2843-3 Morretes, MI-2858-1 Mundo Novo e MI-2858-3 Pedra Branca do Araraquara. Escala 1:50000. SEMA, Curitiba.

ROBIM, M.J., PASTORE, J.A., AGUIAR, O.T. \& BAITELLO, B. 1990. Flora arbóreo arbustiva e herbácea do Parque Estadual de Campos do Jordão (SP). Rev. Inst. Flor. 2:31-53.

ROCHA, M.R.L. 1999. Caracterização fitossociológica e pedológica de uma floresta ombrófila densa no parque estadual do pico do Marumbi - Morretes, PR. Dissertação de Mestrado, Universidade Federal do Paraná, Curitiba.

RODERJAN, C.V. 1994. A floresta ombrófila densa altomontana no morro Anhangava, Quatro Barras, PR: aspectos climáticos, pedológicos e fitossociológicos. Tese de Doutorado, Universidade Federal do Paraná, Curitiba.

RODERJAN, C.V. \& GRODSKI, L. 1999. Acompanhamento meteorológico em um ambiente de Floresta Ombrófila Densa Altomontana no morro Anhangava, Mun. De Quatro Barras - PR, no ano de 1993. Cadernos da Biodiversidade, 2(1):27-34.

RODERJAN, C.V., GALVÃO, F., KUNIYOSHI, Y.S. \& HATSCHBACH, G.G. 2002. As unidades fitogeográficas do estado do Paraná. Cienc. Ambient. 24(1):75-92.

STADTMÜLLER, T. 1987. Cloud Forests in the humid tropics: a bibliographic review. The United Nations University; Centro Agronomico Tropical de Investigacion y Ensenanza, Tokyo; Turrialba.

THOMAS, W.W., CARVALHO, A.M.V., AMORIM, A.M.A., GARRISON, J. \& ARBELÁEZ, A.L. 1998. Plant endemism in two forests in southern Bahia, Brasil. Biodiv. Conserv. 7:311-322.

TRYON, R.M. \& TRYON, A.F. 1982. Ferns and allied plants. Springer Verlag, New York.

VALENTIN, J.L. 2000. Ecologia numérica: uma introdução à análise multivariada de dados ecológicos. Interciência, Rio de Janeiro.

VELOSO, H.P., RANGEL FILHO, A.L.R. \& LIMA, J.C.A. 1991. Classificação da vegetação brasileira, adaptada a um sistema universal. IBGE, Rio de Janeiro.

VITOUSEK, P.M. 1998. Introduction: the structure and functioning of Montane Tropical Forests: Control by climate, soils and disturbance, special feature. Ecology, 79(1):1-2.

Recebido em 12/08/08

Versão Reformulada recebida em 23/03/09

Publicado em 01/04/09 